\title{
On sharing the benefits of communication
}

\author{
Citation for published version (APA):
}

Athanasiou, E., Dey, S., \& Valletta, G. (2012). On sharing the benefits of communication. METEOR, Maastricht University School of Business and Economics. METEOR Research Memorandum No. 016 https://doi.org/10.26481/umamet.2012016

Document status and date:

Published: 01/01/2012

DOI:

10.26481/umamet.2012016

Document Version:

Publisher's PDF, also known as Version of record

\section{Please check the document version of this publication:}

- A submitted manuscript is the version of the article upon submission and before peer-review. There can be important differences between the submitted version and the official published version of record.

People interested in the research are advised to contact the author for the final version of the publication, or visit the DOI to the publisher's website.

- The final author version and the galley proof are versions of the publication after peer review.

- The final published version features the final layout of the paper including the volume, issue and page numbers.

Link to publication

\footnotetext{
General rights rights.

- You may freely distribute the URL identifying the publication in the public portal. please follow below link for the End User Agreement:

www.umlib.nl/taverne-license

Take down policy

If you believe that this document breaches copyright please contact us at:

repository@maastrichtuniversity.nl

providing details and we will investigate your claim.
}

Copyright and moral rights for the publications made accessible in the public portal are retained by the authors and/or other copyright owners and it is a condition of accessing publications that users recognise and abide by the legal requirements associated with these

- Users may download and print one copy of any publication from the public portal for the purpose of private study or research.

- You may not further distribute the material or use it for any profit-making activity or commercial gain

If the publication is distributed under the terms of Article $25 \mathrm{fa}$ of the Dutch Copyright Act, indicated by the "Taverne" license above, 


\section{Maastricht University}

Efthymios Athanasiou, Santanu Dey, Giacomo Valletta

On Sharing the Benefits of Communication

RM/12/016

\section{METEOR}

Maastricht University School of Business and Economics

Maastricht Research School of Economics

of Technology and Organization

P. B. Box 616

NL -6200 MD Maastricht

The Netherlands 


\title{
ON SHARING THE BENEFITS OF COMMUNICATION
}

\author{
Efthymios Athanasiou* ${ }^{*}$, Santanu Dey $^{\dagger}$ and Giacomo Valletta ${ }^{\ddagger}$
}

\begin{abstract}
We put forward a model of private goods with externalities. Agents derive benefit from communicating with each other. In order to communicate they need to have a language in common. Learning languages is costly. In this setting no individually rational and feasible Groves mechanism exists. We characterize the best-in-class feasible Groves mechanism and the best-in-class individually rational Groves mechanism.
\end{abstract}

JEL classification: D70; D62; C60.

Keywords: Groves mechanisms, Externality, Budget surplus or deficit, Pareto undominated mechanisms.

\section{INTRODUCTION}

An agent is associated with one of two platforms. Communication between two agents requires that they operate on a common platform. Adoption of a new platform is costly. The cost depends on the agent's native platform. The benefit of communication depends on a subjective parameter reflecting the value the agent attaches to the collaborative enterprises she may engage in by adopting a new platform. The benefit of communication is increasing in the number of agents one may interact with.

A variety of situations fits this paradigm. Historically, traveling by train between France and Spain required switching trains at the border. In 2010 the completion of an alternative high speed line, operating on the french standard, solved the break of gauge problem. The development of the DVD was made possible only when Philips and Sony abandoned the "Multimedia Compact Disc" format and joined the camp of Toshiba, Time Warner, Pioneer and others developing the "Super Density Disc" format. Finally, to cite a case involving numerous agents, communication among individuals belonging to different language groups in places such as Belgium, Canada and Florida makes necessary the adoption of a foreign language.

The model we propose is the simplest one that adheres to these observations. We build on Selten and Pool [21]. Henceforth, we will use the term language to denote a mode of operation and the term communication to denote the possibilities of interaction that speaking a common language affords. Refer to figure 1. Agents are represented by nodes. The set of agents is partitioned in two language groups. Agent $j$ speaks language $\alpha$ natively. Learning is depicted by an arrow stemming from a node and pointing to a set of nodes. Individual $j$ learns language $\beta$. This enables her to communicate with two agents speaking $\beta$. The benefit each agents derives from communication is given by the number of 'foreign' agents she communicates with, multiplied by a non-negative real number that encompasses the agent's willingness to

We thank Claude d'Aspremont, Marc Fleurbaey, Michele Lombardi, Francois Maniquet, Herve Moulin, Eve Ramaekers, Yves Sprumont, William Thomson, participants at seminars or conferences at the University of Mannheim, Carnegie Mellon University, University of Maastricht, Social Choice and Welfare Conference 2008, 17th Coalition Theory Network Workshop for discussions, comments, or suggestions.

*Dep. of Philosophy, CMU, USA.

${ }^{\dagger}$ ISYE, Georgia Tech , USA.

${ }^{\ddagger}$ Dep. of Economics, Maastricht , The Netherlands. 


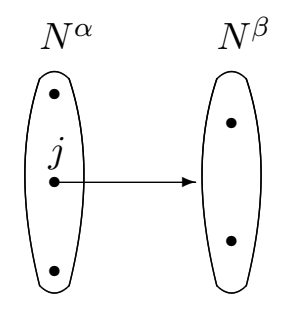

Figure 1. The model

communicate. However learning is costly. The cost agent $j$ faces is determined by the language group she belongs to. The agent's net benefit is the difference between the benefit and the cost of communication. This brief description of the model is sufficient to reveal the nature of an externality that crucially influences the analysis that ensues. Agent $j$, by learning the foreign language $\beta$, affects the well-being of all agents in $N^{\beta}$, even those who choose not learn. Consequently, even agents who are not learning a foreign language are a potential source of value.

The model depicted in figure 1 will be the backdrop against which we will formulate our discussion. We are interested in mechanisms. Roughly speaking, these objects associate a social outcome to the various values the primitives of the model may take. In particular, our main concern will be to examine the extent to which the following four properties can be attributed to a mechanism:

(1) Assignment Efficiency : the sum of net benefits should be maximized.

(2) Strategy-Profness : all agents, if asked, should have a dominant strategy to reveal their willingness to communicate truthfully.

(3) Individual Rationality : no agent should enjoy a level of well-being that is lower than the level of well-being she would enjoy if she was not a constituent of the economy.

(4) Feasibility : the mechanism should rely exclusively on the resources generated within the economy, i.e., no outside funding should be permitted.

It turns out that no mechanism satisfies all of the above requirements. There are, however, mechanisms that satisfy any three of the properties above. We place particular emphasis on Assignment Efficiency and Strategy-Profness. Both these properties are shown to be inherently linked with incentives. A mechanism that violates them is prone to deficiencies that undermine the implementation exercise in a fundamental way.

Appealing to a result due to Holmström [13], embracing Assignment Efficiency and StrategyProofness entails confining our investigation to the family of Groves mechanisms. The literature discussing such mechanisms does so in three fairly distinct contexts. Pure public goods, excludable public goods and private goods.

In this paper we study Groves mechanisms in a context of private goods that accounts for the effect of an externality. It turns out that much of the conventional wisdom on Groves mechanisms does not carry through to our model. An instance of this phenomenon is the impossibility stated above. When all agents value the good positively (as is the case in our framework), the celebrated Pivotal mechanism constitutes an example of a Groves mechanism 
that satisfies the aforementioned properties whether the context is private or public goods ${ }^{1}$. More surprisingly, the Pivotal mechanism here fails Feasibility. The nature of the externality we capture in our model causes the Pivotal mechanism to sometimes assign positive transfers, something that is disallowed in the framework of either public or private goods.

Our proposal involves two mechanisms. First we look at individually rational Groves mechanisms. We show that such mechanisms are often in deficit. We characterize the mechanism that minimizes the deficit whenever it occurs. Second, we single out the only feasible Groves mechanism that is not Pareto dominated by another strategy-proof, feasible and anonymous mechanism. In order to do so we restrict our attention to economies comprising two agents. Both our proposals involve rules that do not appear elsewhere in the literature and are thus specific to the model we put forward.

Effectively the objective we pursue is to identify the best-in-class mechanism. When feasibility is out of the picture the criterion that isolates the best mechanism is related to the incidence of the deficit. We do not focus on the worst case scenario (as in Bailey [2], Cavallo [3], Guo and Conitzer [10], Moulin [18] ) or on the asymptotic behavior of the deficit (as in Deb Razzolini and Seo [6], Green and Laffont [9], McAfee [14] and Zhou [24]). Rather we propose a mechanism that runs a lower deficit than any other mechanism in each economy where the deficit presents itself.

In order to isolate the best feasible Groves mechanism, we do not base our selection on sums of utilities but rather on their distribution (as in Guo and Conitzer [11], Athanasiou [1] and Sprumont [22]). A mechanism Pareto dominates another one if the former generates, in each economy and for each agent, a higher amount of utility. This criterion turns out to be sharp enough to select a unique feasible Groves mechanism when the discussion is confined to two-agent economies.

The analysis is complemented by a discussion of the discrete nature of the problem. In a separate section, we provide an algorithm that identifies efficient linguistic assignments. Interestingly, at the optimum learning by agents of both linguistic groups (two-sided learning) may ensue. Moreover, efficient linguistic assignments do not necessarily impose full communication among agents. At the optimum it may be the case that two agents do not have a language in common.

Aside from the agenda we pursue in this paper, a parallel literature deals with decentralized outcomes that may arise in situations similar to the ones we explore. In their seminal contribution Selten and Pool [21] introduce a general model of language acquisition. They show that an equilibrium of the multi-country multilingual language acquisition model exists. The characterization of an equilibrium is then studied by Church and King [4]. More recently, Ginsburgh et al. [8] and Gabszewicz et al. [7] study qualitative properties of such equilibria in the context of bilingual societies. In our model one may rationalize different Nash Equilibria, exhibiting both one-sided as well as multi-sided learning. However, the efficient outcome does not generically come about as a Nash Equilibrium.

Section 2 introduces the model. Section 3 discusses efficiency. Section 4 introduces the axioms and presents the impossibility. Section 5 discusses individually rational Groves mechanism. Section 6 discusses feasible Groves mechanisms. Section 7 concludes.

\footnotetext{
${ }^{1}$ Parkes [20] provides sufficient conditions for a Pivotal mechanism to be individually rational in a pure public good framework. Moulin [18], among others, makes the case in the context of private goods.
} 


\section{THE MODEL}

The finite set of agents is denoted $N \subseteq \mathbb{N}$. There are two languages, $\alpha$ and $\beta$. Each agent speaks natively one of them. For each $\lambda \in\{\alpha, \beta\}, N^{\lambda}$ is the set of agents speaking language $\lambda$. Each agent may learn the language that is foreign to her. For instance, if $i \in N^{\alpha}$, agent $i$ may choose to either learn language $\beta$ or not. Learning is represented by the dichotomous variable $l_{i} \in\{0,1\}$. The values are $l_{i}=1$ if she learns, $l_{i}=0$ otherwise. Let $l_{N}=\left(l_{i}\right)_{i \in N} \in\{0,1\}^{N}$ denote the vector describing the actions taken by each member of the population. The function $\gamma: N \rightarrow\{\alpha, \beta\}$ such that for each $i \in N$ and for each $\lambda \in\{\alpha, \beta\}$,

$$
\gamma(i)=\lambda \Leftrightarrow i \in N^{\lambda}
$$

determines the native language of each agent. Consequently, for each $i \in N, N^{\gamma(i)}$ denotes the set of agents whose native language is the same as $i$ 's. The cost of learning depends on the agents native language. Let $C=\left(c_{\alpha}, c_{\beta}\right) \in \mathbb{R}_{++}^{2}$. For each $i \in N, c_{\gamma(i)}$ is the learning cost agent $i$, whose native language is $\gamma(i)$, faces. We assume that $c_{\alpha}, c_{\beta}<\infty$.

The benefit an agent derives from being able to communicate with agents from the other language group is a linear function of the number of agents she communicates with at a given assignment $l_{N} \in\{0,1\}^{N}$. The willingness to communicate is denoted by the parameter $\theta_{i} \in \mathbb{R}_{+}$. Hence, for each $i \in N$, at each $l_{N} \in\{0,1\}^{N}$, the expression

$$
\theta_{i} \sum_{j \in N \backslash N^{\gamma(i)}} \min \left\{1,\left(1, l_{i}\right) \cdot\left(l_{j}, 1\right)\right\}
$$

specifies the gross benefit of agent $i$. Let $\theta_{N^{\alpha}} \equiv\left(\theta_{i}\right)_{i \in N^{\alpha}}, \theta_{N^{\beta}} \equiv\left(\theta_{i}\right)_{i \in N^{\beta}}$ and $\theta_{N} \equiv\left(\theta_{i}\right)_{i \in N}$. An economy is denoted by $e=\left(\left(\theta_{N^{\alpha}}, \theta_{N^{\beta}}\right), C\right)=\left(\theta_{N}, C\right) \in \mathbb{R}_{+}^{N+2} \equiv \mathcal{E}$.

The definition of the gross benefit incorporates certain assumptions. The linearity assumption facilitates the exposition. Assuming a decreasing marginal benefit from communication would not alter the nature of our results. It would, therefore, merely complicate derivations without bringing any particular insight.

In our model agents either learn a language or not. That is, we do not allow for degrees of knowledge. All our results would hold if we were to qualify this assumption. In fact, any mathematical difficulty the model presents would remain, even if we were to apply a convexification, namely letting $l_{i}$ take values in an interval, say $[0,1]$. The discrete nature of the problem stems from the min operator (equation 2.1). This operator ensures that even if two agents have two languages in common, the benefit each derives from communicating with the other remains unchanged relative to the case of communication through a single language. Therefore, even if the parameter $l_{i}$ was continuous, the problem of maximizing social welfare would have to be solved under multiple constraints.

The gross benefit does not account for people with whom one shares the same mother tongue. This value is a constant. Therefore, it does not have any bearing in any individual or social maximization.

Finally, agents do not care neither with whom they communicate, nor in what language they do so. For instance, let $N=\{1,2,3,4\}$ with $N^{\alpha}=\{1,2\}$ and $N^{\beta}=\{3,4\}$. Indeed, as long as $l_{3}=l_{4},\left(1, l_{1}\right)\left(l_{3}, 1\right)=\left(1, l_{1}\right)\left(l_{4}, 1\right)$. That is, communicating with agent 3 provides agent 1 with the same (gross) benefit she obtains by communicating with agent 4 . Moreover, no agent may serve as a translator, that is, communication is only possible through the direct adoption of a foreign language. These assumptions, although unrealistic, serve to intensify the effect of the 
externality. Since our aim is precisely to study this effect, we have no interest in dampening its intensity.

For each $i \in N$, the net benefit associated with $l_{N}=\left(l_{i}\right)_{i \in N}$ is

$$
v_{i}\left(l_{N} ; \theta_{i}\right)=\theta_{i} \sum_{j \in N \backslash N^{\gamma(i)}} \min \left\{1,\left(1, l_{i}\right) \cdot\left(l_{j}, 1\right)\right\}-l_{i} c_{\gamma(i)}
$$

For each $e \in \mathcal{E}$ and each $l_{N} \in\{0,1\}^{N}$, let $\pi\left(l_{N} ; e\right)=\sum_{i \in N} v_{i}\left(l_{N} ; \theta_{i}\right)$ be the sum of net benefits generated by the linguistic assignment $l_{N}$.

Accounting for the possibility of an individual transfer $t_{i} \in \mathbb{R}$, the final utility of each agent, at $l_{N} \in\{0,1\}^{N}$, becomes

$$
u_{i}\left(l_{N}, t_{i} ; \theta_{i}\right)=v_{i}\left(l_{N} ; \theta_{i}\right)+t_{i} .
$$

Preferences are quasi-linear. Let $t_{N}=\left(t_{i}\right)_{i \in N} \in \mathbb{R}^{N}$. An allocation is a list $\left(l_{N}, t_{N}\right) \equiv\left(l_{i}, t_{i}\right)_{i \in N}$ where $l_{i}$ is a linguistic assignment for agent $i$ and $t_{i}$ is the transfer she receives. Let $Z$ be the set of all allocations. A mechanism is a function $\varphi$ defined over $\mathcal{E}$ that associates with each economy an allocation $\left(l_{N}, t_{N}\right) \in Z$. Namely

$$
\varphi: \mathcal{E} \rightarrow\{0,1\}^{N} \times \mathbb{R}^{N}=Z,
$$

so that $\varphi(e)=\left(l_{N}, t_{N}\right)$ and $\varphi_{i}(e)=\left(l_{i}, t_{i}\right)$.

\section{EFFICIENT LINGUISTIC ASSIGNMENTS}

In this section we discuss the problem of determining efficient linguistic assignments, that is, assignments that maximize the sum of net benefits. Depending on the particular $e \in \mathcal{E}$ at hand, we need to solve the following optimization problem:

$$
P(e): \max _{l_{N} \in\{0,1\}^{N}} \sum_{i \in N}\left(\theta_{i}\left(\sum_{j \in N / N^{\gamma(i)}} \min \left\{1,\left(1, l_{i}\right) \cdot\left(l_{j}, 1\right)\right\}\right)-l_{i} c_{\gamma(i)}\right) .
$$

For each $e \in \mathcal{E}$, let $\Sigma(e)$ be the set of linguistic assignments that solve $P(e)$. In what follows we provide an algorithm that produces for each $e \in \mathcal{E}$, one $l_{N} \in \Sigma(e)$.

Each agent has at most 2 alternatives, she either learns the other language or she does not. There are $N$ agents. Therefore, there are at most $2^{N}$ candidate solutions. Since the set of candidate solutions is finite, for each $e \in \mathcal{E}, \Sigma(e) \neq \emptyset$.

A naive algorithm for determining efficient linguistic assignment is to enumerate all the candidate solutions. However, such an algorithm is not very efficient since its running time increases exponentially with the increase in the number of agents in the economy. We next present a polynomial time algorithm for obtaining an efficient linguistic assignment, that is, an algorithm whose running time increases as a polynomial function with the increase in the number of agents in the economy.

The construction of the algorithm is founded on Lemmata 1, 2 and 3 presented below. These constitute a few properties of an optimal solution, which allows pruning the search to a small subset of candidates solutions without missing at least one optimal solution.

Lemma 1 states that if at the optimum an agent does not learn, then so do all other agents in her language group that have a lower willingness to communicate.

Lemma 1. For each $e \in \mathcal{E}$, each $\lambda \in\{\alpha, \beta\}$ and each $i_{1}, i_{2} \in N^{\lambda}$, if $\theta_{i_{1}}>\theta_{i_{2}}$, then for each $l_{N} \in \Sigma(e)$ either

(1) $l_{i_{1}}=l_{i_{2}}=0$, or 
(2) if $l_{i_{2}}=1$, then $l_{i_{1}}=1$.

Proof. Without loss of generality let $\lambda=\alpha$. Suppose, by way of contradiction, that there exists $l_{N} \in \Sigma(e)$ such that $l_{i_{1}}=0$ and $l_{i_{2}}=1$. Construct an alternative solution $\tilde{l}_{N}$ such that

$$
\tilde{l}_{j}= \begin{cases}l_{j} & \text { if } j \neq i_{1}, j \neq i_{2} \\ 1 & \text { if } j=i_{1} \\ 0 & \text { if } j=i_{2}\end{cases}
$$

Let also $S \subseteq N^{\beta}$, be such that for each $i \in S, l_{i}=\widetilde{l}_{i}=0$. By construction, $\pi\left(\tilde{l}_{N} ; e\right)-\pi\left(l_{N} ; e\right)$ $=\left(\theta_{i_{1}}-\theta_{i_{2}}\right)|S|$. By assumption, $l_{N}$ is optimal and $l_{i_{2}}=1$, with $i_{2} \in N^{\alpha}$. If $S=\emptyset$ then, for each $i \in N^{\beta}, l_{i}=1$. But if every agent in $N^{\beta}$ learned, it would be suboptimal for agent $i_{2}$ to learn too. Therefore, $S \neq \emptyset$. This implies that $\left(\theta_{i_{1}}-\theta_{i_{2}}\right)|S|>0$, the desired contradiction.

Consider some arbitrary linguistic assignment $l_{N} \in\{0,1\}^{N}$ such that for some $k \in N^{\alpha}$, $l_{k}=0$. If agent $k$ were to learn, she would both attain a personal utility gain equal to $\theta_{k}\left(\left|N \backslash N^{\gamma(k)}\right|-\sum_{j \in N \backslash N^{\gamma(k)}} l_{j}\right)-c_{\gamma(i)}$, namely, her marginal willingness to communicate times the number of agents from the other language group who have not learned $k$ 's language $\left(j \in N^{\beta}\right.$ for whom $l_{j}=0$ ), and benefit these very same agents who would be able to communicate with one more agent. Therefore, agent $k$, by learning would generate a marginal contribution equal to

$$
\theta_{k}\left(\left|N \backslash N^{\gamma(k)}\right|-\sum_{j \in N \backslash N^{\gamma(k)}} l_{j}\right)+\sum_{j \in N \backslash N^{\gamma(k)}} \theta_{j}\left(1-l_{j}\right)-c_{\gamma(k)} .
$$

The following Lemma states two familiar conditions necessary for optimality.

Lemma 2. For each $e \in \mathcal{E}$, each $l_{N} \in \Sigma(e)$ and each $i \in N$,

(1) if $l_{i}=1$, then $\theta_{i}\left(\left|N \backslash N^{\gamma(i)}\right|-\sum_{j \in N \backslash N^{\gamma(i)}} l_{j}\right)+\sum_{j \in N \backslash N^{\gamma(i)}} \theta_{j}\left(1-l_{j}\right)-c_{\gamma(i)} \geq 0$,

(2) if $l_{i}=0$, then $\theta_{i}\left(\left|N \backslash N^{\gamma(i)}\right|-\sum_{j \in N \backslash N^{\gamma(i)}} l_{j}\right)+\sum_{j \in N \backslash N^{\gamma(i)}} \theta_{j}\left(1-l_{j}\right)-c_{\gamma(i)} \leq 0$.

Proof. Conditions (1) and (2) are necessary for $l_{N} \in \Sigma(e)$. Indeed, if $l_{N} \in \Sigma(e)$, then for each $i \in N$ unilaterally reducing the amount of communication (first condition), or unilaterally increasing the amount of communication (second condition) must decrease the sum of utilities.

Finally, we present a consequence of the above two Lemmata.

Lemma 3. For each $e \in \mathcal{E}$, each $\lambda \in\{\alpha, \beta\}$ and each $i^{*} \in N^{\lambda}$ such that $\theta_{i^{*}} \geq \theta_{i}$ for each $i \in N^{\lambda}$, if $l_{N} \in \Sigma(e)$ and $l_{i^{*}}=0$, then

$$
\begin{aligned}
& l_{i}=0 \quad \text { for all } i \in N^{\lambda} \\
& l_{i}= \begin{cases}1 & \text { if }\left|N^{\lambda}\right| \theta_{i}+\sum_{j \in N^{\lambda}} \theta_{j}-c_{\gamma(i)}>0 \quad \text { for all } i \in N \backslash N^{\lambda} \\
0 & \text { otherwise }\end{cases}
\end{aligned}
$$

Proof. $\quad$ Implication (3.2) follows from Lemma 1. Implication (3.3) follows from Lemma 2.

The algorithm is presented in Table 1. It relies on Lemmata 1 and 3 . The algorithm performs approximately $\left|N^{\alpha}\right|\left|N^{\beta}\right|$ arithmetic operations ${ }^{2}$. Using this fact it can be shown that

\footnotetext{
${ }^{2}$ The sorting in the first step can be done in approximately $\left|N^{\alpha}\right| \log _{2}\left(\left|N^{\alpha}\right|\right)+\left|N^{\beta}\right| \log _{2}\left(\left|N^{\beta}\right|\right)$ operations.
} 
for suitably large $N$, the algorithm takes less than $\eta|N|^{2}$ operations where $\eta$ is some constant independent of $e$. Thus the proposed algorithm is a polynomial-time algorithm.

On the left part of figure 3 we elaborate on the algorithm. One may think of the successive iterations of the algorithm as a move by each agent in $N^{\alpha}$, starting from the agent with the highest willingness to communicate and proceeding in descending order. Each agent may move in one of two possible directions; she may choose to learn or not to learn. In this vein, any path from the initial node to any of the $m$ terminal nodes corresponds to a candidate solution. For each $k=1, \ldots, m-1$, let $l_{N} \mid k$ denote any such candidate solution. For instance, at $k=3$ we consider the path $\left(l_{1}=1, l_{2}=1, l_{3}=0\right)$ that corresponds to a linguistic assignment $l_{N} \mid 3$ such that $l_{1}\left|3=l_{2}\right| 3=1$, for each $i \in N^{\alpha} \backslash\{1,2\}, l_{i} \mid 3=0$ and for each $i \in N^{\beta}, l_{i} \mid 3=l_{i}^{\prime}$, where $l_{N}^{\prime}$ solves the problem

$$
P\left(\left(\theta_{N^{\alpha} \backslash\{1,2\}}, \theta_{N^{\beta}}\right), C\right) \text { subject to } l_{i}=0 \text {, for each } i \in N^{\alpha} \backslash\{1,2\} \text {. }
$$

Lemma 3 provides us with the calculation that determines $l_{N}^{\prime}$. Finally, $\pi\left(\left(l_{N} \mid 3 ;\left(\theta_{N^{\alpha}}, \theta_{N^{\beta}}\right), C\right)\right)=$ $M_{1}+M_{2}+\pi\left(\left(l_{N}^{\prime} ;\left(\theta_{N^{\alpha} \backslash\{1,2\}}, \theta_{N^{\beta}}\right), C\right)\right)=M_{1}+M_{2}+V_{3}$. The longest path $\left(l_{1}=1, l_{2}=\right.$ $\left.1, \ldots, l_{m-1}=1\right)$ corresponds to the linguistic assignment $l_{N} \mid m-1$, where for each $i \in N^{\alpha}$, $l_{i} \mid m-1=1$ and for each $i \in N^{\beta}, l_{i} \mid(m)=0$ and $\pi\left(\left(l_{N} \mid(m) ;\left(\theta_{N^{\alpha}}, \theta_{N^{\beta}}\right), C\right)\right)=M_{1}+M_{2}+$ $\cdots+M_{m-1}$.

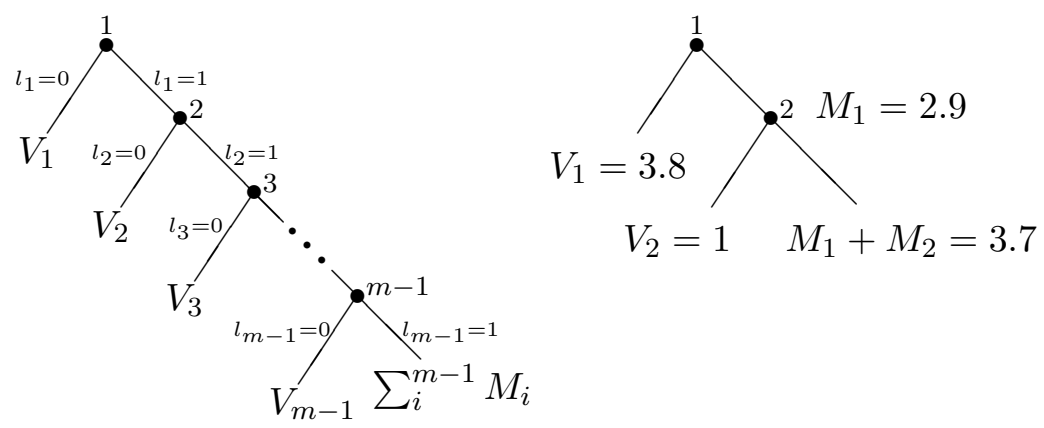

Figure 2

Consider the economy $e=(((0.8,0.1),(2,0.1,0)),(1.6,1.1))$. The algorithm generates three candidate solutions. Refer to the right part of figure 3 . The corresponding values are $V_{1}=3.8$, $V_{2}+M_{1}=3.9$ and $M_{1}+M_{2}=3.7$. The optimal linguistic assignment is $((1,0),(1,0,0))$.

The example above, apart from illustrating how the algorithm works, demonstrates two important points regarding an efficient linguistic assignment. Firstly, at the optimum, full communication does not necessarily ensue. Secondly, and perhaps more surprisingly, optimal linguistic assignments may entail that agents from both language groups learn. 
TABLE 1. The algorithm

(1) Let $N^{\alpha}=\{1, \ldots, m-1\}$ and $N^{\beta}=\{m, \ldots, N\}$. Sort agents so that for each $\lambda \in\{\alpha, \beta\}$ and each $i, j \in N^{\lambda}, i<j$ if and only if $\theta_{i} \geq \theta_{j}$. Let $M_{i}=\left|N \backslash N^{\gamma(i)}\right| \theta_{i}+\sum_{j \in N \backslash N^{\gamma(i)}} \theta_{j}-$ $c_{\gamma(i)}$.

(2) Consider the problem $P\left(\left(\theta_{N^{\alpha}}, \theta_{N^{\beta}}\right), C\right)$.

(a) By Lemma 1 either agent 1 learns $\beta$ or no one in $N^{\alpha}$ learns $\beta$. Therefore, create two subproblems.

(b) In the first subproblem set $l_{1}=0$. By Lemma 1 , for each $i \in N^{\alpha}$ we have $l_{i}=0$. In this case, the optimal solution is obtained using Lemma 3. Call the resulting value $V_{1}$. Save the solution to this subproblem.

(c) In the second subproblem set $l_{1}=1$. This completely determines agent 1 's status. Save the value $M_{1}:=\left|N^{\beta}\right| \theta_{1}+\sum_{j \in N^{\beta}} \theta_{j}-c_{\alpha}$, remove agent 1 from the problem.

(d) Set $t=1$.

(3) If $m-1>t$ go to step (4). Else go to step (5).

(4) The algorithm treats the problem $P\left(\left(\theta_{N^{\alpha} \backslash\{1, \ldots, t\}}, \theta_{N^{\beta}}\right), C\right)$.

(a) By Lemma 1 either agent $t+1$ learns $\beta$ or no one in $N^{\alpha} \backslash\{1, \ldots, t\}$ learns $\beta$. Therefore, create two subproblems.

(b) In the first subproblem set $l_{t+1}=0$. By Lemma 1 , for each $i \in N^{\alpha} \backslash\{1, \ldots, t\}$ we have $l_{i}=0$. In this case, the optimal solution is obtained using Lemma 3. Call the resulting value $V_{t+1}$. Save the solution to this subproblem.

(c) In the second subproblem set $l_{t+1}=1$. This completely determines agent $t+1$ 's status. Save the value $M_{t+1}:=\left|N^{\beta}\right| \theta_{t+1}+\sum_{j \in N^{\beta}} \theta_{j}-c_{\alpha}$, remove agent $t+1$ from the problem.

(d) Set $t=t+1$. Go to (3).

(5) The algorithm generates $m$ solutions. Namely, $V_{1}, V_{2}+M_{1}, V_{3}+M_{1}+M_{2}, \ldots, V_{m-1}+$ $M_{1}+\cdots+M_{m-2}, M_{1}+\cdots+M_{m-1}$. Pick the maximum among them.

\section{Axioms}

In this section we formally define Strategy-Proofness, Assignment Efficiency and Individual Rationality and discuss their implications. Although these axioms may be motivated by appealing to normative considerations, we emphasize here their significance in alleviating the incentive problem. In our framework a mechanism can be manipulated in three ways:

(1) The agent may misreport relevant information she holds private.

(2) The agent may choose not to conform to the prescriptions of the mechanism.

(3) The agent may refuse to participate.

The axioms we present in this section each tackles one of the issues listed above.

Although the social planner observes the partition of agents in language groups and is aware of the costs that learning entails, she does not know the agents' willingness to communicate. As a consequence, some agents might find it profitable do behave strategically and misreport it. We require that mechanism induce for each agent a weakly dominant strategy to report truthfully her willingness to communicate, if asked. 
Strategy - Proofness For each $e \in \mathcal{E}, i \in N$ and $\theta_{i}^{\prime} \in \mathbb{R}_{+}$,

$$
u_{i}\left(\varphi_{i}\left(\theta_{N}, C\right) ; \theta_{i}\right) \geq u_{i}\left(\varphi_{i}\left(\theta_{i}^{\prime}, \theta_{N \backslash\{i\}}, C\right) ; \theta_{i}\right) .
$$

A mechanism is Assignment Efficient if, for each economy in the admissible domain, it selects an allocation that involves a linguistic assignment that maximizes the sum of net benefits. Assignment Efficiency differs from Pareto Efficiency in that it does not require transfers to sum up to zero.

Assignment - Efficiency For each $e \in \mathcal{E}$, if $\left(l_{N}, t_{N}\right)=\varphi(e)$ then $l_{N} \in \Sigma(e)$.

The way Assignment Efficiency wards against manipulation is not immediately apparent. In order to make it explicit we need to emphasize one of the implications of the axiom. Assignment Efficiency implies the following axiom.

Home-Schooling Proofness: For each $e \in \mathcal{E}$, if $\left(l_{N}, t_{N}\right)=\varphi(e)$, then, for each $i \in N$ and $l_{i}^{\prime} \in\{0,1\}$ such that $l_{i}^{\prime}>l_{i}$,

$$
\theta_{i} \sum_{j \in N \backslash N^{\gamma(i)}}\left[\min \left\{1,\left(1, l_{j}\right)\left(1,\left(l_{i}^{\prime}-l_{i}\right)\right)\right\}\right]-\left(l_{i}^{\prime}-l_{i}\right) c_{\gamma(i)}<0 .
$$

Suppose that an allocation is such that Maggie is instructed not to learn. However, given the pattern of language learning that the allocation envisages, she finds it profitable to unilaterally deviate and learn ${ }^{3}$. Even if there is an institution in place that controls whether the agents comply with the instructions of the social planner, it would be very costly to ensure that Maggie sticks to the plan. For instance, it would be hard, indeed almost implausible, to monitor her every step, in order to make sure that her mother, who happens to speak the language, will not home-school her.

A mechanism satisfies Individual Rationality if no agent is coerced into participation. All agents must enjoy a positive utility as a result of their participation.

Individual Rationality For each $e \in \mathcal{E}$ and $i \in N, u_{i}\left(\varphi_{i}(e) ; \theta_{i}\right) \geq 0$.

Since the domain of preference profiles is convex (and hence smoothly connected) we know from Holmstrom [13] that a mechanism satisfies Assignment Efficiency and Strategy Proofness if and only if it belongs to the family of Groves mechanisms (see Groves [12]).

Such mechanisms determine a transfer composed of two parts. First, each agent receives the total net benefit obtained by all other agents at the assignment chosen by the mechanism. Second, each agent receives a sum of money that does not depend on her own (announced) willingness to communicate. Let $h_{i}$ be a real-valued function defined on $\mathbb{R}_{+}^{N-1}$ such that for each $i \in N$ and $\theta_{N} \in \mathbb{R}_{+}^{N}, h_{i}$ depends at most on $\theta_{N \backslash\{i\}}$ and in any case does not depend on agent $i$ 's willingness to communicate.

The Groves Mechanism For each $e \in \mathcal{E},\left(l_{N}, t_{N}\right)=\varphi^{g}(e)$ if and only if $l_{N} \in \Sigma(e)$ and,

\footnotetext{
${ }^{3}$ This could only happen if the linguistic assignment is not efficient.
} 
for each $i \in N$,

$$
t_{i}=\sum_{j \neq i} v_{j}\left(l_{N} ; \theta_{i}\right)-h_{i}\left(\theta_{N \backslash\{i\}}\right)
$$

In general, for Groves mechanisms, the sum of the transfers may be either positive or negative. Generically there is a waste. However, there is a particular difficulty pertaining to a deficit. A Planner will need to finance the Groves scheme using resources that are not generated within the economy. Any mechanism, be it Groves or not, by construction, is silent as to where these outside funds may be found. Feasibility requires that the Planner does not need to resort to outside funding. Feasible mechanisms are self-sufficient.

Feasibility For each $e \in \mathcal{E}$, if $\left(l_{N}, t_{N}\right)=\varphi(e)$ then $\sum_{i \in N} t_{i} \leq 0$.

The exercise we perform in this paper is shaped by the following result. It implies that the Planner may opt for either an individually rational Groves mechanism, at the expense of Feasibility, or a feasible Groves mechanism, at the expense of Individual Rationality.

Proposition 1. There exists no mechanism $\varphi$ that satisfies Strategy Proofness, Individual Rationality, Feasibility and Home Schooling Proofness.

Proof. We construct a counter-example. Suppose that some mechanism $\varphi$ satisfies the axioms. Consider an economy $e$ consisting of two agents, $N=\{1,2\}$, speaking distinct languages. We have $\theta_{1}=\theta_{2}>c_{\alpha}=c_{\beta}>0$ and $c_{\alpha}+c_{\beta}>\theta_{1}, \theta_{2}$. By Home Schooling Proofness, for each $\left(l_{N}, t_{N}\right) \in \varphi(e)$ either one of the following must be true:

(1) $l_{1}=0$ and $l_{2}=0$, or

(2) $l_{1}=0$ and $l_{2}=1$, or

(3) $l_{1}=1$ and $l_{2}=0$.

Consider case (1), i.e. let there exist $\left(l_{N}, t_{N}\right) \in \varphi(e)$ such that $l_{1}=0$ and $l_{2}=0$. Suppose that $u_{1}\left(l_{N}, t_{1} ; \theta_{1}\right) \geq \theta_{1}$ and $u_{2}\left(l_{N}, t_{2} ; \theta_{2}\right) \geq \theta_{2}$. Therefore,

$$
\begin{gathered}
u_{1}\left(l_{N}, t_{1} ; \theta_{1}\right)+u_{2}\left(l_{N}, t_{2} ; \theta_{2}\right) \geq \theta_{2}+\theta_{1} \\
\theta_{1}-c_{\alpha}+\theta_{2}+t_{1}+t_{2} \geq \theta_{1}+\theta_{2} \\
t_{1}+t_{2} \geq c_{\alpha}
\end{gathered}
$$

By Feasibility, $t_{1}+t_{2} \leq 0$ and hence inequality (4.1) constitutes a contradiction, as by assumption $c_{\alpha}>0$. The same reasoning applies for cases (2),(3). In conclusion, for each $\left(l_{N}, t_{N}\right) \in \varphi(e)$, either $u_{1}\left(l_{N}, t_{1} ; \theta_{1}\right)<\theta_{1}$ or $u_{2}\left(l_{N}, t_{2} ; \theta_{2}\right)<\theta_{2}$. Without loss of generality, suppose that for each $\left(l_{N}, t_{N}\right) \in \varphi(e), u_{1}\left(l_{N}, t_{1} ; \theta_{1}\right)<\theta_{1}$.

Consider economy $e^{\prime}$, to be one that is identical to economy $e$, except for the fact that $\theta_{1}^{\prime}=0$. By Individual Rationality and Feasibility, since $c_{\alpha}+c_{\beta}>\theta_{2}$, there cannot exist $\left(l_{N}^{\prime}, t_{N}^{\prime}\right)$ such that $l_{1}^{\prime}=1$ and $l_{2}^{\prime}=1$. Therefore, by Home Schooling Proofness, for each $\left(l_{N}^{\prime}, t_{N}^{\prime}\right) \in \varphi\left(e^{\prime}\right)$, either $l_{1}^{\prime}=1$ and $l_{2}^{\prime}=0$, or $l_{1}^{\prime}=0$ and $l_{2}^{\prime}=1$. By Individual Rationality, in the former case $t_{1}^{\prime} \geq c_{\alpha \beta}$ and in the latter $t_{1}^{\prime} \geq 0$. This implies that from a profile of announcements $\left(\theta_{1}, \theta_{2}\right)$ agent 1 can profitably deviate to the profile $\left(0, \theta_{2}\right)$ and obtain a utility level equal to $\theta_{1}$. Thus, the mechanism $\varphi$ violates Strategy-Proofness, a contradiction. 
Proposition 1 is of interest for an additional reason. It marks a stark difference between our framework and other economic environments where it is well known that feasible and individually rational Groves mechanisms exist. Examples of feasible and individually rational Groves mechanisms are provided by Guo and Conitzer [10] and Moulin [18] (among others) as solutions to the problem of assigning a finite number of identical objects to a greater finite number of agents. When a public good is involved Groves mechanisms are not in general individually rational. Nonetheless, under the assumption that all agents value the good positively, as is the case in our model, the Pivotal mechanism satisfies Individual Rationality. Proposition 1 constitutes, therefore, an early indication suggesting that the conventional wisdom does not straightforwardly apply to our context.

\section{An Individually Rational Groves Mechanism}

In this section we focus on Groves mechanisms that satisfy Individual Rationality. As shown before, such mechanisms violate Feasibility. Among them, we isolate a mechanism that minimizes the deficit whenever it occurs. The natural question to raise next concerns the incidence of the deficit. We provide conditions on the economy that, when met, imply that any Individually Rational Groves mechanism will be in deficit in that economy. Inspection of these sufficient conditions suggest that the deficit is, indeed, a prevalent phenomenon. In fact, running simulations we were not able to find any economy in which an Individually Rational Groves mechanism runs a surplus.

For each $e \in \mathcal{E}$ and each $i \in N$ let $e^{i}$ denote an economy that is otherwise identical to $e$, except for the fact that agent $i$ 's willingness to communicate has been set equal to zero. Formally, if $e=\left(\theta_{N}, C\right)$, then $e^{i}=\left(\left(0, \theta_{N \backslash\{i\}}\right), C\right)$. In addition, let $l_{N}^{i} \in \Sigma\left(e^{i}\right)$.

The Minimal Deficit Mechanism (MDM) For each $e \in \mathcal{E},\left(l_{N}, t_{N}\right)=\varphi^{m d}(e)$ if and only if $l_{N} \in \Sigma(e)$ and, for each $i \in N$,

$$
t_{i}=\sum_{j \neq i} v_{j}\left(l_{N} ; \theta_{i}\right)-\sum_{j \neq i} v_{j}\left(l_{N}^{i} ; \theta_{j}\right)-v_{i}\left(l_{N}^{i} ; 0\right) .
$$

Roughly speaking, the transfer of the MDM constitutes an assessment of the impact each agent's willingness to communicate has on the optimal sum of net benefits. In order to accomplish that, the mechanism, for each agent $i \in N$, needs to calculate $\pi\left(l_{N}^{i} ; e^{i}\right)$. In particular, in order to obtain the MDM from within the family of Groves mechanisms one needs to set, for each $e \in \mathcal{E}$ and each $i \in N$,

$$
h_{i}\left(\theta_{N \backslash\{i\}}\right)=\pi\left(l_{N}^{i} ; e^{i}\right)=\sum_{j \neq i} v_{j}\left(l_{N}^{i} ; \theta_{j}\right)+v_{i}\left(l_{N}^{i} ; 0\right) .
$$

We illustrate the MDM with the help of an example. Consider the economy

$$
e=(((2,1,0.1),(0.8,0.1)),(1.1,1.6)) \text {. }
$$

Refer to figure 3, top left, for a graphical representation. Dots represent agents. They are partitioned in two columns, each representing a language group. Agents whose mother-tongue is $\alpha$ are on the left column, while agents whose mother-tongue is $\beta$ are on the right column. The numbers in parenthesis are the names of the agents. Moreover, $\theta_{1}=2, \theta_{2}=1, \theta_{3}=0.1$, $\theta_{4}=0.8, \theta_{5}=0.1$. The figure depicts also the five economies we obtain by setting, each time, the willingness to communicate of each agent equal to zero. We call these economies $e^{1}, \ldots, e^{5}$. An arrow stemming from a node representing agent $i \in N$ and pointing to a 
language group stands for $l_{i}=1$. The absence of an arrow stands for $l_{i}=0$. The figure depicts $l_{N} \in \Sigma(e)$, as well as $l_{N}^{1}, \ldots, l_{N}^{5}$. For instance, $l_{N}=(0,0,0,1,1)$. Interestingly a slight perturbation in the original problem can drastically change the efficient linguistic assignment as it is made evident by inspecting the figure. Finally, for each $i \in N$, the figure provides $\pi\left(l_{N}^{i} ; e^{i}\right)$ so that it is possible to compute the transfer that the MDM prescribes for $e$, namely, $t_{N}=(-0.2,-0.2,0,0.9,1.6)$. Agents 1 and 2 are taxed, while agents 4 and 5 are subsidized. The MDM produces a deficit equal to 2.1.

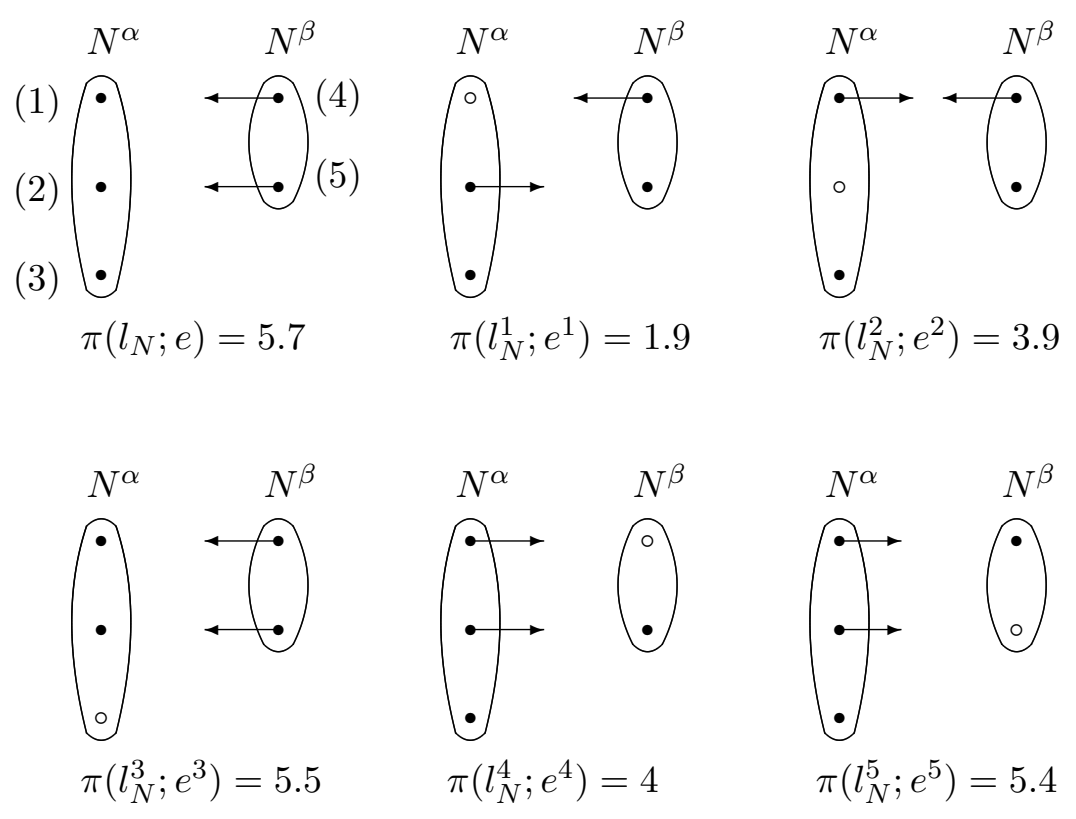

Figure 3. An example

The MDM needs to be distinguished from the Pivotal mechanism (see Clarke [5] and Moulin [16] for a detailed description of the properties of such mechanism in the pure public good framework). In order to obtain the Pivotal mechanism from the family of Groves mechanisms one needs to set, for each $e \in \mathcal{E}$ and each $i \in N$,

$$
h_{i}\left(\theta_{N \backslash\{i\}}\right)=\sum_{j \neq i} v_{j}\left(l_{N \backslash\{i\}}^{\prime} ; \theta_{j}\right),
$$

where $l_{N \backslash\{i\}}^{\prime} \in \Sigma\left(\theta_{N \backslash\{i\}}, C\right)$. The $h_{i}($.$) component of the Pivotal transfer is obtained by$ removing agent $i$ from the economy altogether and then calculating the optimal sum of net benefits in her absence. An apparent difference between the Pivotal mechanism and the MDM is that the former, unlike the latter, by removing the agent from the economy, deprives the remaining agents from any benefit they may derive from being able to communicate with her. In the canonical public good provision model whether an agent is removed from the economy or her valuation of the project is set to zero, amounts to the same effect. In our framework, the nature of the externality that each agent produces on the other agents is different. An agent is still a potential source of value for the rest even if her willingness to communicate is equal to zero. Removing her from the economy amounts to more than deducting her net benefit from the total sum. This fact has stark implications as the following Lemma demonstrates 
Lemma 4. If for some $e \in \mathcal{E}$ the $M D M$ generates a deficit then, in the same economy, the Pivotal mechanism generates a greater or equal deficit.

Proof. By construction, for each $e=\left(\theta_{N}, C\right) \in \mathcal{E}$ and each $l_{N \backslash\{i\}} \in \Sigma\left(\theta_{N \backslash\{i\}}, C\right)$,

$$
\pi\left(\left(l_{N \backslash\{i\}}, 0\right) ; e^{i}\right) \geq \pi\left(l_{N \backslash\{i\}} ;\left(\theta_{N \backslash\{i\}}, C\right)\right) .
$$

If, $l_{N}^{\prime} \in \Sigma\left(e^{i}\right)$, then, by definition,

$$
\pi\left(l_{N}^{\prime} ; e^{i}\right) \geq \pi\left(\left(l_{N \backslash\{i\}}, 0\right) ; e^{i}\right) .
$$

Therefore, for each $e=\left(\theta_{N}, C\right) \in \mathcal{E}$, each $l_{N \backslash\{i\}} \in \Sigma\left(\theta_{N \backslash\{i\}}, C\right)$ and each $l_{N}^{\prime} \in \Sigma\left(e^{i}\right)$,

$$
\pi\left(l_{N}^{\prime} ; e^{i}\right) \geq \pi\left(l_{N \backslash\{i\}} ;\left(\theta_{N \backslash\{i\}}, C\right)\right) .
$$

Let $\varphi^{p}$ denote the Pivotal mechanism. For some $e \in \mathcal{E}$ let $\left(l_{N}^{m d}, t_{N}^{m d}\right)=\varphi^{m d}(e)$ and $\left(l_{N}^{p}, t_{N}^{p}\right)=$ $\varphi^{p}(e)$. By definition, using inequality 5.1, we obtain, for each $i \in N, t_{i}^{m d} \leq t_{i}^{c}$. Therefore,

$$
\sum_{i \in N} t_{i}^{m d} \leq \sum_{i \in N} t_{i}^{p}
$$

In the canonical public good provision model the transfers associated with the Pivotal mechanism are non-positive. It follows from Lemma 4 that this it is no longer the case in our framework. However, both in the canonical public good provision model and our framework the Pivotal mechanism satisfies Individual Rationality. The following proposition generalizes Lemma 4 namely, any individually rational Groves mechanism generates at least as much deficit as the MDM.

Proposition 2. If for some $e \in \mathcal{E}$ the $M D M$ generates a deficit then, in the same economy, any mechanism satisfying Assignment Efficiency, Strategy-Profness and Individual Rationality generates a greater or equal deficit.

Proof. By Assignment Efficiency and Strategy-Proofness we need to compare our mechanism with other mechanisms belonging to the Groves family of mechanisms. Moreover, by Individual Rationality we need to have, for each $e \in \mathcal{E}$ and each $i \in N$,

$$
u_{i}\left(\varphi^{g}(e) ; \theta_{i}\right)=v_{i}\left(l_{N} ; \theta_{i}\right)+t_{i}=\sum_{i \in N} v_{i}\left(l_{N} ; \theta_{i}\right)-h_{i}\left(\theta_{N \backslash\{i\}}\right) \geq 0,
$$

with

$$
\sum_{i \in N} v_{i}\left(l_{N} ; \theta_{i}\right)=\sum_{i \in N}\left(\theta_{i} \sum_{j \in N \backslash N^{\gamma(i)}} \min \left\{1,\left(1, l_{i}\right) \cdot\left(l_{j}, 1\right)\right\}-l_{i} c_{\gamma(i)}\right),
$$

where $l_{N} \in \Sigma(e)$. Hence, for any given profile $\theta_{N \backslash\{i\}} \in \mathbb{R}^{N-1}$ the component $\sum_{i \in N} v_{i}\left(l_{N} ; \theta_{i}\right)$, which is the sum of net benefits at an efficient linguistic assignment, reaches its minimum value when agent $i$ 's willingness to communicate is equal to zero. Hence, in order to satisfy Individual Rationality we need to set, for each $e \in \mathcal{E}, i \in N$,

$$
h_{i}\left(\theta_{N \backslash\{i\}}\right) \leq \sum_{j \neq i} v_{j}\left(l_{N}^{i} ; \theta_{j}\right)+v_{i}\left(l_{N}^{i} ; 0\right)
$$

where $l_{N}^{i} \in \Sigma\left(e^{i}\right)$. Moreover,

$$
\sum_{i \in N} t_{i}=\sum_{i \in N} \sum_{j \neq i} v_{j}\left(l_{N} ; \theta_{j}\right)-\sum_{i \in N} h_{i}\left(\theta_{N \backslash\{i\}}\right)
$$


By equation 5.2,

$$
\sum_{i \in N} t_{i} \geq \sum_{i \in N} \sum_{j \neq i} v_{j}\left(l ; \theta_{j}\right)-\sum_{i \in N} \sum_{j \neq N} v_{j}\left(l^{i} ; \theta_{j}\right)-\sum_{i \in N} v_{i}\left(l_{N}^{i} ; 0\right) .
$$

This means that, as soon an individually rational mechanism generates a deficit, it generates at least as much deficit as in the right-end side of equation 5.3. Hence in order to minimize the deficit produced by the mechanism we need to set

$$
h_{i}\left(\theta_{N \backslash\{i\}}\right)=\sum_{j \neq i} v_{j}\left(l_{N}^{i} ; \theta_{j}\right)+v_{i}\left(l_{N}^{i} ; 0\right) .
$$

We actually conjecture that, for any conceivable economy, any individually rational Groves mechanism runs a deficit. As a consequence the sum of the transfers pertaining to the MDM would always be non-negative as well and. While we are unable to prove the statement in general, first, simulations suggest that it is true and, second, we are able to show it in specific environments. In particular, we prove it to be the case in subdomains of $\mathcal{E}$ that encompass, inter alia, large economies. This is of interest since, as pointed for example by Deb, Razzolini and Seo [6], some members of the Groves family are asymptotically balanced as the number of agents increases in a well-behaved way, for instance by replication. In our framework, if any feasible Groves mechanism is asymptotically balanced, it would need to be the MDM.

The next two propositions state that in a significant sub-domain of the set of admissible economies any Groves mechanism satisfying Individual Rationality runs a deficit. More precisely, Propositions 3 and 4 determine the number of agents that need to have a willingness to communicate greater than some arbitrary non-negative real number $T$ for an individually rational Groves mechanism to run a deficit. The actual value of the threshold $T$ depends on all the parameters of the economy, namely its size, the profile of preferences and costs. Both Propositions assume, without loss of generality, that $c_{\alpha} \leq c_{\beta}$. The proofs can be found in Appendix 1.

For each $e \in \mathcal{E}$ and each $T>0$ let $\zeta(T, e)=\left|\left\{i \in N \mid \theta_{i}<T\right\}\right|$.

Proposition 3. At each $e \in \mathcal{E}$ such that $c_{\alpha} \leq c_{\beta}$ and $\left|N^{\alpha}\right| \leq\left|N^{\beta}\right|$, if there exists $T>0$ such that $\left|N^{\beta}\right| \geq \frac{c_{\alpha}}{T}+\frac{c_{\beta}}{T}+\zeta(T, e)+1$, then any Individually Rational Groves mechanism runs a deficit.

Proposition 4. At each $e \in \mathcal{E}$ such that $c_{\alpha} \leq c_{\beta}$ and $\left|N^{\alpha}\right| \geq \frac{c_{\beta}}{c_{\alpha}}\left|N^{\beta}\right|$, if there exists $T>0$ such that $\left|N^{\alpha}\right| \geq \frac{c_{\beta}}{c_{\alpha}}(\zeta(T, e)+1)+\frac{2 c_{\beta}}{T}$, then any Individually Rational Groves mechanism runs a deficit.

The following examples elaborate on Propositions 3 and 4. Consider the economy

$$
e=(((3,2,1,0),(3,3,2,2,1.5,1.5,0.5)),(1,2)) .
$$

The number of agents with $\theta_{i}$ less that 1 , i.e., $\zeta(1, e)$, is 2 . Hence, $\zeta(1, e)+1+\frac{c_{\alpha}+c_{\beta}}{1}=6<$ $\left|N^{\beta}\right|=7$. By Proposition 3 , in this economy any individually rational Groves mechanism runs a deficit. Consider, next, the economy

$$
e=(((\epsilon, \ldots, \epsilon),(\epsilon, \ldots, \epsilon)),(q \epsilon, q \epsilon))
$$


where $\epsilon>0$ and $q \in \mathbb{N}$. The number of agents with $\theta_{i}$ less that $\epsilon$, i.e., $\zeta(\epsilon, e)$, is equal to zero. Moreover, $\left|N^{\alpha}\right|=\frac{c_{\beta}}{c_{\alpha}}\left|N^{\beta}\right|=\left|N^{\beta}\right|$. We obtain

$$
\frac{q \epsilon}{q \epsilon}(\zeta(\epsilon, e)+1)+\frac{2 q \epsilon}{\epsilon}=1+2 q .
$$

Hence, as soon as $\left|N^{\alpha}\right| \geq 1+2 q$, by Proposition 4, any Individually Rational Groves mechanism runs a deficit.

Two comments are in line. First, the domain restrictions 'almost' do not rely on preferences. In effect, may be interpreted to concern the number of agents who do not value the possibility of communication. Secondly, the domain restrictions do not require the economy to be large, unless both costs are enormous. Even in such a case though, one needs only one language group to be adequately large for the result to come through.

\section{A feasible Groves Mechanism}

In this section we drop Individual Rationality in favor of Feasibility. The restriction to feasible Groves mechanisms reflects a physical constraint often imposed on the implementation effort. The social planner is not mandated to resort to outside funding. She may only rely on her power to tax agents which may be, for the purposes of this section, complemented by her ability to coerce participation.

Given the analysis performed so far, there is a simple class of mechanisms that accomplishes the task. To retrieve it we need to expand on the discussion that ensued in the previous section. In particular, we need to take advantage of a feature of the MDM. For each economy, let the set of linguistic assignments that ensure full communication be denoted $L^{f}(e)$. Consider within this set the subset of linguistic assignments that are the least costly. That is, for each $e \in \mathcal{E}$, let

$$
L^{f *}(e) \equiv \underset{l_{N} \in L^{f}(e)}{\operatorname{argmin}} \sum_{i \in N} l_{i} c_{\gamma(i)} \subseteq L^{f}(e)
$$

Moreover, for each $l_{N}^{*} \in L^{f *}(e)$, let

$$
\underline{c}=\sum_{i \in N} l_{i}^{*} c_{\gamma(i)} .
$$

Notice that in order to compute the value $\underline{c}$ one does not need to know the profile of preferences. It turns out that for each economy the total cost pertaining to the efficient linguistic assignment in that economy will be less or equal to the value $\underline{c}$ for that economy. The mechanism we present below charges all individuals an amount $\frac{c}{N}$ on the top of what they were charged by the MDM.

The Translated Minimal Deficit Mechanism (TMDM) For each $e \in \mathcal{E},\left(l_{N}, t_{N}\right)=\varphi^{t m d}(e)$ if and only if $l_{N} \in \Sigma(e)$ and, for each $i \in N$

$$
t_{i}=\sum_{j \neq i} v_{j}\left(l_{N} ; \theta_{i}\right)-\sum_{j \neq i} v_{j}\left(l_{N}^{i} ; \theta_{j}\right)-v_{i}\left(l_{N}^{i} ; 0\right)-\frac{\underline{c}}{N},
$$

where $l_{N}^{i} \in \Sigma\left(e^{i}\right)$.

Relative to the MDM, the TMDM levies an extra amount $\underline{c}$ that aims at ensuring feasibility. Moreover, the TMDM collects an equal share of this extra amount from each agent. One 
may imagine alternative ways of distributing this extra burden. However, as long as a Groves mechanism levies an amount $\underline{c}$ over the amount the MDM levies, it will satisfy Feasibility. This is a direct consequence of the following Lemma.

Lemma 5. For each $e \in \mathcal{E}$, if $\left(l_{N}, t_{N}\right)=\varphi^{m d}(e)$, then

$$
\sum_{i \in N} t_{i} \leq \sum_{i \in N} l_{i} c_{\gamma(i)} .
$$

Proof. $\quad$ For each $e \in \mathcal{E}$ and each $i \in N$, if $l_{N} \in \Sigma(e)$ and $l_{N}^{i} \in \Sigma\left(e^{i}\right)$, then

$$
\theta_{i} \sum_{j \in N \backslash N^{\gamma(i)}} \min \left\{1,\left(1, l_{i}\right) \cdot\left(l_{j}, 1\right)\right\} \geq \sum_{i \in N} v_{i}\left(l_{N} ; \theta_{i}\right)-\sum_{j \neq i} v_{j}\left(l_{N}^{i} ; \theta_{j}\right)-v_{i}\left(l_{N}^{i} ; 0\right) \text {. }
$$

If that were not true, by rearranging the terms of the inequality one would obtain

$\pi\left(l_{N}^{i} ; e^{i}\right)=\sum_{j \neq i} v_{j}\left(l_{N}^{i} ; \theta_{j}\right)+v_{i}\left(l_{N}^{i} ; 0\right)<\sum_{i \in N} v_{i}\left(l_{N} ; \theta_{i}\right)-\theta_{i} \sum_{j \in N \backslash N^{\gamma(i)}} \min \left\{1,\left(1, l_{i}\right) \cdot\left(l_{j}, 1\right)\right\}=\pi\left(l_{N} ; e^{i}\right)$,

which constitutes a contradiction, as, by assumption, $l_{N}^{i} \in \Sigma\left(e^{i}\right)$. Summing over $i \in N$ we obtain

$$
\sum_{i \in N} \theta_{i} \sum_{j \in N \backslash N^{\gamma(i)}} \min \left\{1,\left(1, l_{i}\right) \cdot\left(l_{j}, 1\right)\right\} \geq N \pi\left(l_{N} ; e\right)-\sum_{i \in N} \pi\left(l_{N}^{i} ; e^{i}\right) .
$$

The left-hand side of the previous equation represents the total gross benefit deriving from communication at $l_{N}$. A simple algebraic manipulation yields

$$
\sum_{i \in N} l_{i} c_{\gamma(i)} \geq(N-1) \pi\left(l_{N} ; e\right)-\sum_{i \in N} \pi\left(l_{N}^{i} ; e^{i}\right)=\sum_{i \in N} t_{i} .
$$

Lemma 5 provides a rough idea of the challenge one needs to overcome when designing feasible Groves mechanisms. If the planner knows for each $e \in \mathcal{E}$, with $\left(l_{N}, t_{N}\right)=\varphi^{m d}(e)$, the value $\sum_{i \in N} l_{i} c_{\gamma(i)}$, then she has at her disposal a rough rule of thumb that she may apply in order to comply with Feasibility. However, Lemma 5 does not do much more than pointing in the right direction. The value $\sum_{i \in N} l_{i} c_{\gamma(i)}$ varies with the economy and there is no a priori reason to be hopeful that collecting the information required to calculate it complies with Strategy-Proofness. The TMDM circumvents this issue by utilizing the fact that for each $e \in \mathcal{E}$, each $l_{N} \in \Sigma(e)$ and each $l_{N}^{\prime} \in L^{f *}(e)$, we have $\sum_{i \in N} l_{i} c_{\gamma(i)} \geq \sum_{i \in N} l_{i}^{\prime} c_{\gamma(i)}=\underline{c}$.

At first glance there is nothing outright opposable with the TMDM. It does violate Individual Rationality, but this is a concession we knew we had to make. Nonetheless, the fact that the TMDM relies on $\underline{c}$, a value insensitive to changes in individual preferences, makes the TMDM suffer from a fundamental flaw. It is Pareto dominated by another mechanism that satisfies among other things Strategy-Proofness and Feasibility. This is due to the fact that the TMDM is generated by a translation of the MDM that does not utilize any information on the preferences. Can we do better within the confines of Strategy-Proofness?

To facilitate the analysis we assume that $N^{\alpha}=\{1\}$ and $N^{\beta}=\{2\}$. Moreover, assume that $c=c_{\alpha}<c_{\beta}$, so that if ever learning is efficient, it is individual 1 that learns 'beta'. An economy is denoted $e=\left(\theta_{N}, c\right) \in \mathbb{R}_{+}^{3}$. Figure 6 below depicts the TMDM for this constricted set of economies, if the domain of economies is reduced to the ones comprising two agents the space from which profiles of preferences are drawn becomes the non-negative real orthant. We will adhere to these assumptions for the rest of this section. Although we suspect our analysis extends to the general case, in order to check that one would need to know precisely how the 
efficient linguistic assignment changes with the economy. For two agents this is immediate. The combinatorial nature of the problem makes the acquisition of this piece of information much more difficult in the general case. In what follows we look, in the two agent case, for feasible Groves mechanisms that are Second-Best efficient.

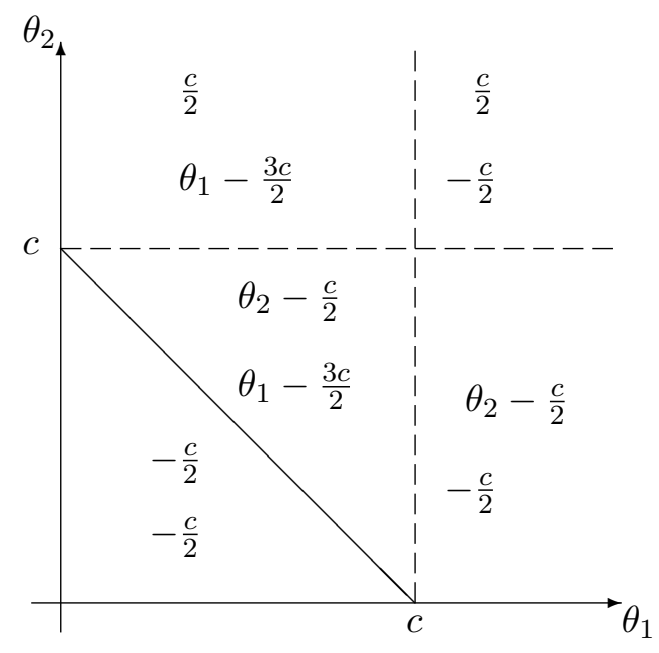

FiguRE 4. The vector of transfers $\left(\begin{array}{c}t_{1}^{t m d} \\ t_{2}^{t m d}\end{array}\right)$ for each profile $\left(\theta_{1}, \theta_{2}\right) \in \mathbb{R}_{+}$according to the TMDM in the two agent case. Agent 1 learns if $\theta_{1}+\theta_{2} \geq 0$. She does not otherwise.

In order to build a notion of Second-Best efficiency we will rely on Anonymity. Let $\tau$ : $\{1,2\} \rightarrow\{1,2\}$ be such that $\tau(i)=j$ for each $i, j \in N$ and $\theta_{\pi(N)}$ denote the vector $\theta_{N}$ permuted according to $\tau$. In the two-agent setting Anonymity effectively requires that the distribution of utility that an allocation induces does not depend on the agent specific cost that learning entails. The axiom constitutes a minimal legitimacy requirement that all mechanisms should naturally fulfill. Moreover, it focuses the exercise we are about to perform. A dictatorial mechanism that always assigns all the surplus to a given individual will be Pareto undominated. A criterion that aims at identifying optimal mechanisms needs to exclude such a perverse phenomenon.

Anomymity For each $e \in \mathcal{E}, i \in N$,

$$
u_{i}\left(\varphi_{i}\left(\theta_{N}, c\right) ; \theta_{i}\right)=u_{\tau(i)}\left(\varphi_{\tau(i)}\left(\theta_{\tau(N)}, c\right)\right)
$$

We may now define the criterion that isolates the best-in-class mechanism. Let $\Phi$ be the set of Strategy-Proof, Anonymous and Feasible mechanisms. For each pair of mechanisms $\varphi, \varphi^{\prime} \in \Phi$, $\varphi$ Pareto dominates $\varphi^{\prime}$ if and only if, for each $e=\left(\theta_{N}, c\right) \in \mathbb{R}_{+}^{3}$, letting $\left(l_{N}, t_{N}\right)=\varphi(e)$ and $\left(l_{N}^{\prime}, t_{N}^{\prime}\right)=\varphi^{\prime}(e)$

for each $i \in N, \quad v_{i}\left(l_{N} ; \theta_{i}\right)+t_{i} \geq v_{i}\left(l_{N}^{\prime} ; \theta_{i}\right)+t_{i}^{\prime}$, and

for some $j \in N, \quad v_{j}\left(l_{N} ; \theta_{j}\right)+t_{j}>v_{j}\left(l_{N}^{\prime} ; \theta_{j}\right)+t_{j}^{\prime}$.

A mechanism $\varphi \in \Phi$ is Second Best Efficient if and only if there does not exist another mechanism $\varphi^{\prime} \in \Phi$ such that $\varphi^{\prime}$ Pareto dominates $\varphi$. Now that we have defined the notion we intend to use to single out the best-in-class mechanism we may go back to the mechanism we 
proposed at the beginning of this section. Clearly the TMDM belongs to $\Phi$ but, is it a Second Best Efficient mechanism? In order to answer this question we provide a simple necessary condition for a mechanism to be Second Best Efficient.

A mechanism $\varphi \in \Phi$ satisfies Condition $\boldsymbol{A}$ if and only if for each $e=\left(\theta_{N}, c\right) \in \mathbb{R}_{+}^{3}$ and each $i \in N$, there does not exist $\epsilon>0$ such that

$$
\begin{gathered}
\left(l_{N}(x), t_{N}(x)\right)=\varphi\left(\left(x, \theta_{N \backslash\{i\}}\right), c\right), \text { and } \\
\text { for each } x \geq 0, \quad \sum_{j \in N} t_{j}(x)+\epsilon \leq 0 .
\end{gathered}
$$

The following proposition states that if a mechanism is such that for some profile of preferences no individual in the economy by unilaterally deviating and changing his announced willingness to communicate can reach a new profile of preferences where the sum of transfers prescribed by the mechanism is arbitrarily close to zero, then the aforementioned mechanism is Pareto dominated. What is more, in order to construct the mechanism that Pareto dominates it, so the proof goes, one needs only perturb the original mechanism ever so slightly.

Proposition 5. A mechanism $\varphi \in \Phi$ is Second Best Efficient only if it satisfies Condition A.

Proof. Suppose that $\varphi \in \Phi$ is Second Best Efficient. By way of contradiction let there exist $e^{\prime}=\left(\theta_{N}^{\prime}, c^{\prime}\right) \in \mathbb{R}_{+}^{3}$ and $\epsilon>0$ such that for some $j \in\{1,2\}$

$$
\begin{gathered}
\left(l_{N}^{\prime}(x), t_{N}^{\prime}(x)\right)=\varphi\left(\left(x, \theta_{N \backslash\{j\}}^{\prime}\right), c^{\prime}\right), \text { and } \\
\quad \text { for each } x \geq 0, \quad \sum_{i \in N} t_{i}^{\prime}(x)+\epsilon \leq 0 .
\end{gathered}
$$

Without loss of generality, let $j=1$. Consider the mechanism $\hat{\varphi}$ constructed in the following way: For each $e=\left(\left(\theta_{1}, \theta_{2}\right), c\right) \in \mathbb{R}_{+}^{3}$ if either $\theta_{1} \neq \theta_{2}^{\prime}$ or $\theta_{2} \neq \theta_{2}^{\prime}$ or $c \neq c^{\prime}$, then $\hat{\varphi}(e)=\varphi(e)$. Otherwise, $\hat{\varphi}\left(\left(x, \theta_{2}^{\prime}\right), c^{\prime}\right)=\left(\hat{l}_{N}(x), \hat{t}_{N}(x)\right)$, where, for each $x \geq 0$,

(i) $\hat{l}_{N}(x)=l_{N}^{\prime}(x)$,

(ii) $\hat{t}_{1}(x)=t_{1}^{\prime}(x)+\epsilon$ and $\hat{t}_{2}(x)=t_{2}^{\prime}(x)$,

Moreover for each $x \geq 0, \hat{\varphi}\left(\left(\theta_{2}^{\prime}, x\right), c^{\prime}\right)=\left(\left(\hat{l}_{2}(x), \hat{l}_{1}(x)\right),\left(\hat{t}_{2}(x), \hat{t}_{1}(x)\right)\right)$. By assumption, $\varphi$ satisfies Strategy-Proofness and Anonymity. Hence, by construction, so does $\hat{\varphi}$. By assumption, $\varphi$ satisfies Feasibilty. Hence, since the negation of Condition $A$ is true, by construction, so does $\hat{\varphi}$. Therefore, $\hat{\varphi} \in \Phi$. By construction, for each $e \in \mathcal{E}$ and each $i \in\{1,2\}$,

$$
u_{i}\left(\hat{\varphi}(e) ; \theta_{i}\right) \geq u_{i}\left(\varphi(e) ; \theta_{i}\right) \text {. }
$$

Moreover, by construction, for each $x \geq 0$,

$$
u_{1}\left(\hat{\varphi}\left(\left(x, \theta_{2}^{\prime}\right), c^{\prime}\right) ; \theta_{j}\right)>u_{1}\left(\varphi\left(\left(x, \theta_{2}^{\prime}\right), c^{\prime}\right) ; \theta_{j}\right) .
$$

Therefore, $\varphi$ cannot be Second Best Efficient, a contradiction.

Refer to figure 4 . It can be readily verified that the TMDM does not satisfy Condition A. Indeed let us fix $\theta_{1}$ so that $\theta_{1}=\theta_{1}^{\prime}<c$. The value of the sum of the transfers prescribed by the TMDM then only depend on $\theta_{2}$. Compute it, for each $\theta_{2} \geq 0$. It is easy to check that $\sum_{j \in N} t_{j}^{t m d}\left(\theta_{2}\right)<0$. Moreover, the fact that the latter inequality is strict provides the intuition behind Proposition 5. In fact, it must be possible to build a mechanism $\hat{\varphi}\left(\left(\theta_{1}^{\prime}, x\right), c\right)=$ $\left(\hat{l}_{N}(x), \hat{t}_{N}(x)\right)$, in such a way that, for each $x \geq 0, \hat{l}_{N}(x)=l_{N}^{t m d}(x), \hat{t}_{1}(x)=t_{1}^{t m d}(x)$ and 
$\hat{t}_{2}(x)=t_{2}^{t m d}(x)+\epsilon$, is still feasible, for some $\epsilon>0$ and, by construction, Pareto dominates $\varphi^{t m d}$. Moreover, Proposition 5 can be generalized to the $N$-agent case where it provides a useful tool to rule out mechanisms that are Pareto dominated by some other mechanism.

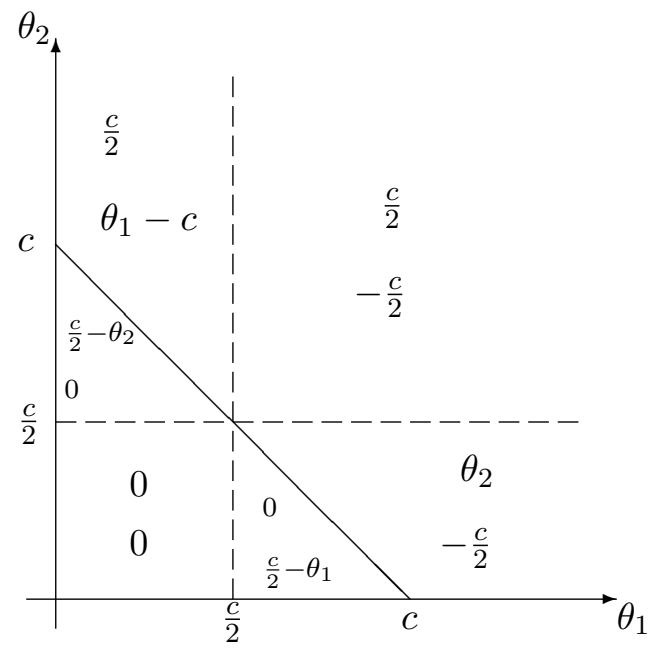

FigURE 5. The vector of transfers $\left(\begin{array}{c}t_{1}^{s b m} \\ t_{2}^{s b m}\end{array}\right)$ for each profile $\left(\theta_{1}, \theta_{2}\right) \in \mathbb{R}_{+}$according to the SBM in the two agent case. Agent 1 learns if $\theta_{1}+\theta_{2} \geq 0$. She does not otherwise.

Interestingly, in the two-agent setting, by ruling out all the Pareto dominated mechanisms we are left with only one Anonymous and Feasible Groves mechanism. For each $c \in \mathbb{R}_{+}$figure 5 defines a Feasible mechanism, call it the Second Best Mechanism, or SBM. The mechanism violates Individual Rationality at each $\left(\theta_{N}, c\right) \in \mathcal{E}$ such that either $\theta_{1}<c$ or $\theta_{2}<c$.

Proposition 6. A mechanism satisfying Strategy-Proofness, Anonymity and Assignment Efficiency is Second Best Efficient if and only if it is the SBM.

The proof can be found in Appendix 2. The subtlety of Proposition 6 becomes apparent by comparing the SBM with the TMDM. The Pareto criterion does not rank the two. The TMDM is preferred to the SBM by agent 1 in economy $e=\left(\theta_{N}, c\right) \in \mathbb{R}_{+}^{3}$ such that $\theta_{1} \in\left(0, \frac{c}{2}\right)$, $\theta_{2} \in\left(\frac{c}{2}, c\right)$ and $\theta_{1}+\theta_{2}>c$. The mechanism that Pareto dominates the TMDM, hence rendering it not Second Best Efficient, must be itself not Second Best Efficient.

\section{Concluding Remarks}

This paper focuses on Groves mechanisms in a model of private goods with externalities. Agents need a common language in order to communicate and the procedure that allows them to acquire such a knowledge is costly. We first provide an algorithm that allows to single out the set of efficient linguistic assignments (i.e., that maximize the sum of net benefits that agents derive from communication) and then we tackle the issue of implementing one of such assignments by mean of a Groves mechanism.

The externality present in the problem radically changes the characteristics of well-known solutions like the Pivotal Mechanism that, in our framework, is no longer feasible. Indeed we show that there is no Groves mechanism that is both Individually Rational and Feasible. This fact forces us to explore two distinct venues. 
We first look at Groves mechanisms that are Individually Rational and among them we single out the mechanism that minimizes the amount of money necessary to finance it. We also provide quite general conditions under which an Individually Rational Groves mechanism needs to resort on outside funding.

We then look at feasible Groves mechanisms. Even if it is relatively simple to find examples of such mechanisms it proves to be an extremely more demanding task to single out mechanisms that are Pareto Undominated. By focusing on the simpler but still meaningful domain of economies comprising only two agents we are able to single out the only second best efficient and feasible Groves mechanism.

Even if the paper focuses solely on Groves mechanisms, it is worth making the point that there are interesting mechanisms not belonging to this class that merit further investigation (see for example Moulin and Shenker [15] or Moulin [17]). To keep things simple let us maintain the simplifying assumptions we made in the previous section. Consider the following simple mechanism defined over two agent economies depicted in figure 6 .

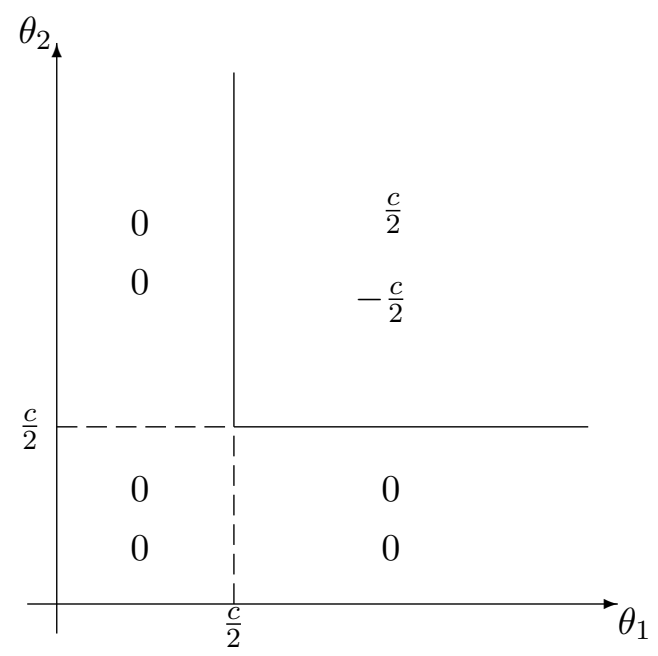

Figure 6 . The vector of transfers $\left(\begin{array}{l}t_{1} \\ t_{2}\end{array}\right)$ for each profile $\left(\theta_{1}, \theta_{2}\right) \in \mathbb{R}_{+}$. Agent 1 learns if $\theta_{1} \geq \frac{c}{2}$ or $\theta_{2} \geq \frac{c}{2}$. She does not otherwise.

Effectively, the mechanism asks agents to announce their willingness to communicate. If the announcements are such that either $\theta_{1}<\frac{c}{2}$ or $\theta_{2}<\frac{c}{2}$ there is no learning and each agent receives a transfer equal to zero. Otherwise, agent 1 learns and $t_{1}=\frac{c}{2}=-t_{2}$. This simple mechanism satisfies Strategy-Proofness, Individual Rationality and Feasibility (in fact, the sum of transfers equals zero in all two-agent economies). Alas, it violates Home-Schooling Proofness and, thus, Assignment Efficiency. To see that, suppose that $\theta_{2}=0<c<\theta_{1}$. The mechanism prescribes to agent 1 to refrain from learning. Nonetheless, agent 1 can ensure a higher utility if he deviates from the proposed allocation and learn. Aside from this deficiency, one should not be hasty in dismissing the mechanism depicted in figure 4. Applying the reasoning employed in the proof of Proposition 5, it can be demonstrated that it is Second Best Efficient. This fact alludes to the existence of interesting mechanisms outside the Groves family and it is left for future research. 


\section{REFERENCES}

[1] Athanasiou, E., 2011. A Solomonic Solution to the Problem of Assigning a Private Indivisible Object, mimeo.

[2] Bailey, M.J., 1997. The demand revealing process: To distribute the surplus, Public Choice 91, $107 Đ 126$

[3] R. Cavallo, R., 2006. Optimal decision-making with minimal waste: Strategyproof redistribution of VCG payments. International Conference on Autonomous Agents and Multi-agents Systems, (AAMAS) Hakodate, Japan.

[4] Church, J., King I., 1993. Bilingualism and network externalities. Canadian Journal of Economics 26, 337-345.

[5] Clarke, E., 1971. Multipart pricing of public goods. Public Choice 8, 19-33.

[6] Deb, R., Razzolini, L., Seo T.K., 2006. The conservative equal cost rule, the serial cost sharing rule and the pivotal mechanism: asymptotic welfare loss comparisons for the case of an excludable public project. Review of Economic Design 10, 205-232.

[7] Gabszewicz J., Ginsburgh V., Weber S. 2005, Bilingualism and Communicative Benefits, Annales d'Economie et de Statistique, forthcoming.

[8] Ginsburgh V., Ortuno-Ortin I., Weber S., 2006, Learning Foreign Languages. Theoretical Implications of the Selten and Pool Model, European Economic Review, 64, 337-347.

[9] J. Green, J., Laffont J.J., 1979. Incentives in Public Decision Making, North-Holland, Amsterdam.

[10] Guo M., Conitzer V., 2007. Worst case optimal redistribution of VCG payments. Conference on Electronic Commerce (EC), San Diego.

[11] Guo M., Conitzer V., 2008. Undominated VCG redistribution mechanisms. Proceedings of the $7^{\text {th }}$ International Conference on Autonomous Agents and Multiagent Systems (AAMAS-08), Estoril, Portugal, 1039-1046.

[12] Groves, T., 1973. Incentives in teams. Econometrica 41, 617-631.

[13] Holmström, B., 1979. Groves' scheme on restricted domains. Econometrica 47, 1137-1144.

[14] McAfee, R.P., 1992. A dominant strategy double auction, J. Econ. Theory 56, 434Đ450

[15] Moulin, H., Shenker S., 1992. Serial cost sharing. Econometrica 50, 1009-1039.

[16] Moulin, H., 1986. Characterizations of the Pivotal Mechanism. Journal of Public Economics 31, 53-78.

[17] Moulin, H., 1994. Serial Cost-Sharing of Excludable Public Goods. Review of Economic Studies 61, 305-25.

[18] Moulin, H., 2009. Almost budget-balanced VCG mechanisms to assign multiple objects, Journal of Economic Theory 144, 96-119.

[19] Nisan, N., Roughgarden, T., Tardos, E., Vaziriani, V., 2009. Algorithmic game theory, Cambridge University Press, New York, USA.

[20] Parkes, D.C., 2001. Classic Mechanism Design. Iterative Combinatorial Auctions: Achieving Economic and Computational Efficiency, Ph.D. dissertation, Univesity of Pennsylvania.

[21] Selten, R. and Pool, J., 1991, The distribution of foreign language skills as a game equilibrium, in R. Selten, ed., Game Equilibrium Models, vol. 4, Berlin: Springer-Verlag, 64-84.

[22] Sprumont, Y., 2012. On optimal strategy-proof assignment. Mimeo.

[23] Thomson, W., 2006, Strategy-proof resource allocation rules. Mimeo.

[24] Zhou, L., 2007. The failure of Groves mechanisms in canonical allocation models, Mimeo, Arizona State University.

\section{Appendix 1. Proofs of Propositions 3 and 4}

By inspecting the proof of Proposition 2 (namely the lower bound set by inequality 5.3) the following fact can be easily deduced. Let $\varphi$ be an individually rational Groves mechanism. For each $e=\left(\theta_{N}, C\right) \in \mathcal{E}$, with $\left(l_{N}, t_{N}\right)=\varphi(e)$, if

$$
\sum_{i \in N} \sum_{j \neq i} v_{j}\left(l_{N} ; \theta_{j}\right)-\sum_{i \in N} \sum_{j \in N} v_{j}\left(l_{N}^{i} ; \theta_{j}\right)-\sum_{i \in N} v_{i}\left(l_{N}^{i} ; 0\right)=(|N|-1) \pi\left(l_{N}, e\right)-\sum_{i \in N} \pi\left(l_{N}^{i}, e^{i}\right) \geq 0
$$

then

$$
\sum_{i \in N} t_{i} \geq 0
$$


In what follows we assume without loss of generality that $c_{\alpha} \leq c_{\beta}$, that $N^{\alpha}=\{1, \ldots, m-1\}$, $N^{\beta}=\{m, \ldots,|N|\}$ and that, for each $\lambda \in\{\alpha, \beta\}$ and for each $i, j \in N^{\lambda}$, if $i<j$, then $\theta_{i} \geq \theta_{j}$.

Throughout his section $l_{N} \in \Sigma(e)$ and $l_{N}^{i} \in \Sigma\left(e^{i}\right)$. Moreover, some times it will be more convenient to denote $l_{N}$ representing explicitly the agents that actually learn at such a linguistic assignment. So, for each $e \in \mathcal{E}$ and $l_{N} \in \Sigma(e)$ let $I\left(l_{N} ; e\right)=\left\{i \in N^{\alpha} \mid l_{i}=1\right\}$ and $J\left(l_{N} ; e\right)=\{i \in$ $\left.N^{\beta} \mid l_{i}=1\right\}$. With a slight abuse of notation we will just write $I\left(l_{N} ; e\right)=I$ and $J\left(l_{N} ; e\right)=J$ so that $(I, J)$ is the set of agents who learn a foreign language at some efficient assignment $l_{N}$. Similarly, some times it will be more convenient to denote $l_{N}^{i}$ as $\left(I^{i}, J^{i}\right)$. For any $\tilde{I} \subseteq N^{\alpha}$ and $\tilde{J} \subseteq N^{\beta}$ we use $z(\tilde{I}, \tilde{J})$ to denote the value of such a structure in the economy $e$, i.e.,

$$
z(\tilde{I}, \tilde{J})=\left|N^{\beta}\right| \sum_{i \in \tilde{I}} \theta_{i}+|\tilde{I}| \sum_{j \in N^{\beta} \backslash \tilde{J}} \theta_{j}+\left|N^{\alpha}\right| \sum_{j \in \tilde{J}} \theta_{j}+|\tilde{J}| \sum_{i \in N^{\alpha} \backslash \tilde{I}} \theta_{i}-|\tilde{I}| c_{\alpha}-|\tilde{J}| c_{\beta}
$$

Finally similar to $z(\tilde{I}, \tilde{J})$, for any $\tilde{I} \subseteq N^{\alpha}$ and $\tilde{J} \subseteq N^{\beta}$ we use $z^{i}(\tilde{I}, \tilde{J})$ to denote the value of such a structure in the economy $e^{i}$.

The following Lemmata are needed for the proofs of Propositions 3 and 4.

Lemma 6. For each $i \in N^{\alpha}, \pi\left(l_{N}^{i} ; e^{i}\right) \leq \pi\left(l_{N}^{1} ; e^{1}\right)+\left|N^{\beta}\right|\left(\theta_{1}-\theta_{i}\right)$ and for each $i \in N^{\beta}$, $\pi\left(l_{N}^{i} ; e^{i}\right) \leq \pi\left(l_{N}^{m} ; e^{m}\right)+\left|N^{\alpha}\right|\left(\theta_{m}-\theta_{i}\right)$.

Proof. Let $\left(I^{i}, J^{i}\right)$ be an optimal solution yielding a total surplus of $\pi\left(l_{N}^{i} ; e^{i}\right)$. There are three cases:

(1) $i \in I^{i}$ : Since in $e^{i}$ we set $\theta_{i}$ to zero, this implies that, by Lemma $1, I^{i}=N^{\alpha}$ and $J^{i}=\emptyset$. Therefore,

which implies

$$
\pi\left(l_{N}^{i} ; e^{i}\right)-z^{1}\left(N^{\alpha}, \emptyset\right)=-\left|N^{\beta}\right| \theta_{i}+\left|N^{\beta}\right| \theta_{1}
$$

$$
\pi\left(l_{N}^{i} ; e^{i}\right)=z^{1}\left(N^{\alpha}, \emptyset\right)+\left|N^{\beta}\right|\left(\theta_{1}-\theta_{i}\right) \leq \pi\left(l_{N}^{1} ; e^{1}\right)+\left|N^{\beta}\right|\left(\theta_{1}-\theta_{i}\right) .
$$

(2) $i \notin I^{i}, 1 \in I^{i}$ : Then

$$
\pi\left(l_{N}^{i} ; e^{i}\right)=\left|N^{\beta}\right| \sum_{p \in I^{i}} \theta_{p}+\left|N^{\alpha}\right| \sum_{j \in J^{i}} \theta_{j}+\left|I^{i}\right| \sum_{j \in N^{\beta} \backslash J^{i}} \theta_{j}+\left|J^{i}\right| \sum_{p \in N^{\alpha} \backslash\left(I^{i} \cup i\right)} \theta_{p}-\left|I^{i}\right| c_{\alpha}-\left|J^{i}\right| c_{\beta} .
$$

Moreover

$$
\begin{aligned}
& z^{1}\left(\left(I^{i} \backslash\{1\}\right) \cup\{i\}, J^{i}\right)=\left|N^{\beta}\right| \sum_{p \in\left(I^{i} \backslash 1 \cup\{i\}\right)} \theta_{p}+\left|N^{\alpha}\right| \sum_{j \in J^{i}} \theta_{j}+\left|I^{i}\right| \sum_{j \in N^{\beta} \backslash J^{i}} \theta_{j} \\
& +\left|J^{i}\right| \sum_{p \in N^{\alpha} \backslash\left(\left(\left(I^{i} \backslash\{1\}\right) \cup\{i\}\right) \cup\{1\}\right)} \theta_{p}-\left|I^{i}\right| c_{\alpha}-\left|J^{i}\right| c_{\beta} .
\end{aligned}
$$

Therefore,

$$
\pi\left(l_{N}^{i} ; e^{i}\right)-z^{1}\left(\left(I^{i} \backslash\{1\}\right) \cup\{i\}, J^{i}\right)=-\left|N^{\beta}\right| \theta_{i}+\left|N^{\beta}\right| \theta_{1} .
$$

Therefore,

$$
\pi\left(l_{N}^{i} ; e^{i}\right) \leq \pi\left(l_{N}^{1} ; e^{1}\right)+\left|N^{\beta}\right|\left(\theta_{1}-\theta_{i}\right) .
$$

(3) $i \notin I^{i}, 1 \notin I^{i}$ : Then, by Lemma $3, I^{i}=\emptyset$. So,

$$
\pi\left(l_{N}^{i} ; e^{i}\right)=\left|N^{\alpha}\right| \sum_{j \in J^{i}} \theta_{j}+\left|J^{i}\right| \sum_{p \in N^{\alpha} \backslash\{i\}} \theta_{p}-\left|J^{i}\right| c_{\beta} .
$$


Moreover,

$$
z^{1}\left(\emptyset, J^{i}\right)=\left|N^{\alpha}\right| \sum_{j \in J^{i}} \theta_{j}+\left|J^{i}\right| \sum_{p \in N^{\alpha} \backslash\{1\}} \theta_{p}-\left|J^{i}\right| c_{\beta}
$$

Therefore

$$
\pi\left(l_{N}^{i} ; e^{i}\right)-z^{1}\left(\emptyset, J^{i}\right)=\left|J^{i}\right|\left(\theta_{1}-\theta_{i}\right)
$$

Therefore,

$$
\pi\left(l_{N}^{i} ; e^{i}\right) \leq \pi\left(l_{N}^{1} ; e^{1}\right)+\left|J^{i}\right|\left(\theta_{1}-\theta_{i}\right) \leq \pi\left(l_{N}^{1} ; e^{1}\right)+\left|N^{\beta}\right|\left(\theta_{1}-\theta_{i}\right) .
$$

The same argument may be employed for the proof of the second part of the statement.

Lemma 7. If $1 \notin I^{1}$ then, for each $i \in N^{\alpha}, z\left(I^{1} \cup\{1\}, J^{1}\right)-\pi\left(l_{N}^{i} ; e^{i}\right) \geq\left|N^{\beta}\right| \theta_{i}+\sum_{j \in N^{\beta} \backslash J^{1}} \theta_{j}-$ $c_{\alpha}$. If $m \notin J^{m}$ then, for each $i \in N^{\beta}, z\left(I^{m}, J^{m} \cup\{m\}\right)-\pi\left(l_{N}^{i} ; e^{i}\right) \geq\left|N^{\alpha}\right| \theta_{i}+\sum_{j \in N^{\alpha} \backslash I^{m}} \theta_{j}-c_{\beta}$.

Proof. $\quad$ If $1 \notin I^{1}$ then, by definition, $z\left(I^{1} \cup\{1\}, J^{1}\right)-\pi\left(l_{N}^{1} ; e^{1}\right)=\left|N^{\beta}\right| \theta_{1}+\sum_{j \in N^{\beta} \backslash J^{1}} \theta_{j}-c_{\alpha}$. Moreover from Lemma 6 we have that, for each $i \in N^{\alpha}, \pi\left(l_{N}^{i} ; e^{i}\right) \leq \pi\left(l_{N}^{1} ; e^{1}\right)+\left|N^{\beta}\right| \theta_{1}-\left|N^{\beta}\right| \theta_{i}$ and therefore $z\left(I^{1} \cup\{1\}, J^{1}\right)-\pi\left(l_{N}^{i} ; e^{i}\right) \geq\left|N^{\beta}\right| \theta_{i}+\sum_{j \in N^{\beta} \backslash J^{1}} \theta_{j}-c_{\alpha}$. Similarly for the second part of the statement.

An immediate consequence of Lemma 7 is that, If $1 \notin I^{1}$, then $\sum_{i \in \tilde{I}}\left(\pi\left(l_{N} ; e\right)-\pi\left(l_{N}^{j} ; e^{i}\right)\right) \geq$ $\sum_{i \in \tilde{I}}\left(z\left(I^{1} \cup\{1\}, J^{1}\right)-\pi\left(l_{N}^{i} ; e^{i}\right)\right) \geq\left|N^{\beta}\right| \sum_{i \in \tilde{I}} \theta_{i}+|\tilde{I}| \sum_{j \in N^{\beta} \backslash J^{1}} \theta_{j}-|\tilde{I}| c_{\alpha}$ where $\tilde{I}$ is any subset of $N^{\alpha}$. Similarly, if $m \notin J^{m}$, then $\sum_{j \in \tilde{J}}\left(\pi\left(l_{N} ; e\right)-\pi\left(l_{N}^{j} ; e^{j}\right)\right) \geq \sum_{j \in \tilde{J}}\left(z\left(I^{m}, J^{m} \cup\{m\}\right)-\right.$ $\left.\pi\left(l_{N}^{j} ; e^{j}\right)\right) \geq\left|N^{\alpha}\right| \sum_{j \in \tilde{J}} \theta_{j}+|\tilde{J}| \sum_{i \in N^{\alpha} \backslash I^{m}} \theta_{i}-|\tilde{J}| c_{\beta}$ where $\tilde{J}$ is any subset of $N^{\beta}$.

Lemma 8. If either $I^{1}=N^{\alpha}$ or $J^{m}=N^{\beta}$, then any Individually Rational Groves mechanism runs a deficit.

Proof. Suppose that $I^{1}=N^{\alpha}$. By Lemma 6 we have that for each $i \in N^{\alpha}, \pi\left(l_{N}^{i} ; e^{i}\right) \leq$ $\pi\left(l_{N}^{1} ; e^{1}\right)+\left|N^{\alpha}\right|\left(\theta_{1}-\theta_{i}\right)$. Moreover, since by assumption $\left(I^{1}, J^{1}\right)=\left(N^{\alpha}, \emptyset\right)$, we have that for each $i \in N^{\alpha}, z^{i}\left(N^{\alpha}, \emptyset\right)=\pi\left(l_{N}^{1} ; e^{1}\right)+\left|N^{\alpha}\right|\left(\theta_{1}-\theta_{i}\right)$. Combining the two statements we obtain that for each $i \in N^{\alpha}, \pi\left(l_{N}^{i} ; e^{i}\right) \leq z^{i}\left(N^{\alpha}, \emptyset\right)$. By definition, $l_{N}^{i} \in \Sigma\left(e^{i}\right)$, therefore, for each $i \in N^{\alpha}, \pi\left(l_{N}^{i} ; e^{i}\right)=z^{i}\left(N^{\alpha}, \emptyset\right)$. In turn, this latter fact implies that for each $i \in N^{\alpha}$, $\pi\left(l_{N}^{i} ; e\right)-\pi\left(l_{N}^{i} ; e^{i}\right)=\left|N^{\beta}\right| \theta_{i}$. Moreover, since $l_{N} \in \Sigma(e)$, we have that for each $i \in N^{\alpha}$, $\pi\left(l_{N} ; e\right) \geq \pi\left(l_{N}^{i} ; e\right)$. Therefore, combining the last two steps we obtain

$$
\sum_{i \in N^{\alpha}}\left(\pi\left(l_{N} ; e\right)-\pi\left(l_{N}^{i} ; e^{i}\right)\right) \geq\left|N^{\beta}\right| \sum_{i \in N^{\alpha}} \theta_{i} .
$$

Claim: There exists an optimal linguistic assignment for $e^{m},\left(I^{m}, J^{m}\right)$, such that $m \notin J^{m}$. Suppose, by way of contradiction, that at all the optimal linguistic assignments for the economy $e^{m}, m \in J^{m}$. Then, by Lemma 1 , at any such linguistic assignment we must have $J^{m}=N^{\beta}$. Therefore, $z^{m}\left(N^{\beta}, \emptyset\right) \geq z^{m}\left(\emptyset, N^{\alpha}\right)$, which is true only if $\left|N^{\alpha}\right| c_{\alpha} \geq\left|N^{\beta}\right| c_{\beta}$. Moreover, since $I^{1}=N^{\alpha}$, by applying the same reasoning as before, we have that $\left|N^{\alpha}\right| c_{\alpha} \leq\left|N^{\beta}\right| c_{\beta}$. Thus, $\left|N^{\alpha}\right| c_{\alpha}=\left|N^{\beta}\right| c_{\beta}$ and therefore the solution $I^{m}=N^{\alpha}, J^{m}=\emptyset$ is also optimal, a contradiction. We may therefore apply Lemma 7. It yields

$$
\sum_{j \in J^{m} \cup\{m\}}\left(z\left(I^{m}, J^{m} \cup\{m\}\right)-\pi\left(l_{N}^{j} ; e^{j}\right)\right) \geq
$$




$$
\left|N^{\alpha}\right| \sum_{j \in\left(J^{m} \cup\{m\}\right)} \theta_{j}+\left|J^{m} \cup\{m\}\right| \sum_{i \in N^{\alpha} \backslash I^{m}} \theta_{i}-\left|J^{m} \cup\{m\}\right| c_{\beta} .
$$

Adding (8.3) and (8.4) we obtain

$$
\begin{aligned}
& \sum_{i \in N^{\alpha}}\left(\pi\left(l_{N} ; e\right)-\pi\left(l_{N}^{i} ; e^{i}\right)\right)+\sum_{j \in J^{m} \cup\{m\}}\left(z\left(I^{m}, J^{m} \cup\{m\}\right)-\pi\left(l_{N}^{j} ; e^{j}\right)\right) \geq\left|N^{\beta}\right| \sum_{i \in N^{\alpha}} \theta_{i} \\
& +\left|N^{\alpha}\right| \sum_{j \in\left(J^{m} \cup\{m\}\right)} \theta_{j}+\left|J^{m} \cup\{m\}\right| \sum_{i \in N^{\alpha} \backslash I^{m}} \theta_{i}-\left|J^{m} \cup\{m\}\right| c_{\beta} .
\end{aligned}
$$

Case 1. Let $I^{m}=N^{\alpha}$. By Lemma 6 we have that for each $i \in N^{\beta}, \pi\left(l_{N}^{i} ; e^{i}\right) \leq \pi\left(l_{N}^{m} ; e^{m}\right)+$ $\left|N^{\alpha}\right|\left(\theta_{m}-\theta_{i}\right)$. Moreover, since by assumption $\left(I^{m}, J^{m}\right)=\left(N^{\alpha}, \emptyset\right)$, we have that for each $i \in N^{\beta}, z^{i}\left(N^{\alpha}, \emptyset\right)=\pi\left(l_{N}^{m} ; e^{m}\right)+\left|N^{\beta}\right|\left(\theta_{m}-\theta_{i}\right)$. Combining the two statements we obtain that for each $i \in N^{\beta}, \pi\left(l_{N}^{i} ; e^{i}\right) \leq z^{i}\left(N^{\alpha}, \emptyset\right)$. By definition, $l_{N}^{i} \in \Sigma\left(e^{i}\right)$, therefore, for each $i \in N^{\beta}, \pi\left(l_{N}^{i} ; e^{i}\right)=z^{i}\left(N^{\alpha}, \emptyset\right)$. In turn, this latter fact implies that for each $i \in N^{\beta}, \pi\left(l_{N}^{i} ; e\right)-$ $\pi\left(l_{N}^{i} ; e^{i}\right)=\left|N^{\alpha}\right| \theta_{i}$. Moreover, since $l_{N} \in \Sigma(e)$, we have that for each $i \in N^{\beta}, \pi\left(l_{N} ; e\right) \geq$ $\pi\left(l_{N}^{i} ; e\right)$. Therefore, combining the last two steps we obtain

$$
\sum_{i \in N^{\beta}}\left(\pi\left(l_{N} ; e\right)-\pi\left(l_{N}^{i} ; e^{i}\right)\right) \geq\left|N^{\alpha}\right| \sum_{i \in N^{\beta}} \theta_{i} .
$$

Combining (8.3) and (8.6) we obtain

$$
|N| \pi\left(l_{N} ; e\right)-\sum_{i \in N} \pi\left(l_{N}^{i} ; e^{i}\right) \geq\left|N^{\beta}\right| \sum_{i \in N^{\alpha}} \theta_{i}+\left|N^{\alpha}\right| \sum_{i \in N^{\beta}} \theta_{i}
$$

Clearly, $\left|N^{\beta}\right| \sum_{i \in N^{\alpha}} \theta_{i}+\left|N^{\alpha}\right| \sum_{i \in N^{\beta}} \theta_{i}>\pi\left(l_{N} ; e\right)$, so that inequality (8.7) becomes inequality (8.1). This completes the proof for this case.

Case 2. Let $I^{m} \subset N^{\alpha}$. Therefore, the set $N^{\alpha} \backslash I^{m}$ is not empty. At the optimal solution corresponding to $e^{m},\left(I^{m}, J^{m}\right)$, by resorting to Lemma 3, we obtain that for each $i \in N^{\alpha} \backslash I^{m}$, $\theta_{i}+\sum_{j \in N^{\beta} \backslash J^{m} \cup\{m\}} \theta_{j}-c_{\alpha} \leq 0$. As $\theta_{i} \geq 0$, we get $\sum_{j \in N^{\beta} \backslash\left(J^{m} \cup\{m\}\right)} \theta_{j}-c_{\alpha} \leq 0$.

By using this latter observation (8.5) becomes

$$
\begin{aligned}
& \sum_{i \in N^{\alpha}}\left(\pi\left(l_{N} ; e\right)-\pi\left(l_{N}^{i} ; e^{i}\right)\right)+\sum_{j \in J^{m} \cup\{m\}}\left(z\left(I^{m}, J^{m} \cup\{m\}\right)-\pi\left(l_{N}^{j} ; e^{j}\right)\right. \\
& \geq\left|N^{\beta}\right| \sum_{i \in N^{\alpha}} \theta_{i}+\left|N^{\alpha}\right| \sum_{j \in\left(J^{m} \cup\{m\}\right)} \theta_{j}+\left|J^{m} \cup\{m\}\right| \sum_{i \in N^{\alpha} \backslash I^{m}} \theta_{i} \\
& -\left|J^{m} \cup\{m\}\right| c_{\beta}+\left|I^{m}\right| \sum_{j \in N^{\beta} \backslash\left(J^{m} \cup\{m\}\right)} \theta_{j}-\left|I^{m}\right| c_{\alpha} \\
& =\left|N^{\beta}\right| \sum_{i \in N_{\alpha} \backslash I^{m}} \theta_{i}+z\left(I^{m}, J^{m} \cup\{m\}\right) \geq z\left(I^{m}, J^{m} \cup\{m\}\right) .
\end{aligned}
$$

Therefore, we obtain that

$$
\left.\sum_{i \in N^{\alpha}}\left(\pi\left(l_{N} ; e\right)-\pi\left(l_{N}^{i} ; e^{i}\right)\right)+\left|J^{m}\right| z\left(I^{m}, J^{m} \cup\{m\}\right)-\sum_{j \in J^{m} \cup\{m\}} \pi\left(l_{N}^{j} ; e^{j}\right)\right) \geq 0 .
$$

Since $\pi\left(l_{N} ; e\right) \geq z\left(I^{m}, J^{m} \cup\{m\}\right)$, for each $j \in N, \pi\left(l_{N} ; e\right) \geq \pi\left(l_{N}^{j} ; e^{j}\right)$ and by assumption $\left|J^{m}\right| \leq\left|N^{\beta}\right|-1$ we obtain that $\left(\left|N^{\alpha}\right|+\left|N^{\beta}\right|-1\right) \pi\left(l_{N} ; e\right)-\sum_{i \in N^{\alpha}} \pi\left(l_{N}^{i} ; e^{i}\right)-\sum_{j \in N^{\beta}} \pi\left(l_{N}^{j} ; e^{j}\right) \geq 0$, or, put differently, inequality (8.1). This completes the proof for this case. 
The same argument may be employed to prove the statement when $J^{m}=N^{\beta}$.

Lemma 9. If $1 \notin I^{1}, m \notin J^{m}$ and either $I^{1} \cup\{1\} \supseteq I^{m}$ or $J^{m} \cup\{m\} \supseteq J^{1}$ then any Individually Rational Groves mechanism runs a deficit.

Proof. $\quad$ Since $1 \notin I^{1}$ and $m \notin J^{m}$ then, by Lemma 7,

$$
\begin{aligned}
& \sum_{i \in I^{1} \cup\{1\}}\left(z\left(I^{1} \cup\{1\}, J^{1}\right)-\pi\left(l_{N}^{i} ; e^{i}\right)\right)+\sum_{i \in J^{1}}\left(z\left(I^{m}, J^{m} \cup\{m\}-\pi\left(l_{N}^{j} ; e^{j}\right)\right)\right) \\
& \geq\left|N^{\beta}\right| \sum_{i \in\left(I^{1} \cup\{1\}\right)} \theta_{i}+\left|I^{1} \cup\{1\}\right| \sum_{j \in N^{\beta} \backslash J^{1}} \theta_{j}-\left|I^{1} \cup\{1\}\right| c_{\alpha} \\
& +\left|N^{\alpha}\right| \sum_{j \in J^{1}} \theta_{j}+\left|J^{1}\right| \sum_{j \in N^{\alpha} \backslash I^{m}} \theta_{i}-\left|J^{1}\right| c_{\beta} .
\end{aligned}
$$

Since $I^{1} \cup\{1\} \supseteq I^{m}$, then $N^{\alpha} \backslash I^{m} \supseteq N^{\alpha} \backslash\left(I^{1} \cup\{1\}\right)$. Thus $\left|J^{1}\right| \sum_{j \in N^{\alpha} \backslash I^{m}} \theta_{i} \geq\left|J^{1}\right| \sum_{N^{\alpha} \backslash\left(I^{1} \cup\{1\}\right)} \theta_{i}$. Therefore, we may rewrite (8.9) as follows,

$$
\begin{aligned}
& \sum_{i \in I^{1} \cup\{1\}}\left(z\left(I^{1} \cup\{1\}, J^{1}\right)-\pi\left(l_{N}^{i} ; e^{i}\right)\right)+\sum_{i \in J^{1}}\left(z\left(I^{m}, J^{m} \cup\{m\}\right)-\pi\left(l_{N}^{j} ; e^{j}\right)\right) \\
\geq & \left|N^{\beta}\right| \sum_{i \in\left(I^{1} \cup\{1\}\right)} \theta_{i}+\left|I^{1} \cup\{1\}\right| \sum_{j \in N^{\beta} \backslash J^{1}} \theta_{j}-\left|I^{1} \cup\{1\}\right| c_{\alpha} \\
+ & \left|N^{\alpha}\right| \sum_{j \in J^{1}} \theta_{j}+\left|J^{1}\right| \sum_{j \in N^{\alpha} \backslash I^{m}} \theta_{i}-\left|J^{1}\right| c_{\beta} \\
\geq & \left|N^{\beta}\right| \sum_{i \in\left(I^{1} \cup\{1\}\right)} \theta_{i}+\left|I^{1} \cup\{1\}\right| \sum_{j \in N^{\beta} \backslash J^{1}} \theta_{j}-\left|I^{1} \cup\{1\}\right| c_{\alpha} \\
+ & \left|N^{\alpha}\right| \sum_{j \in J^{1}} \theta_{j}+\left|J^{1}\right| \sum_{j \in N^{\alpha} \backslash\left(I^{1} \cup\{1\}\right)} \theta_{i}-\left|J^{1}\right| c_{\beta} \\
= & z\left(I^{1} \cup\{1\}, J^{1}\right) .
\end{aligned}
$$

Now, since $\pi\left(l_{N} ; e\right) \geq z\left(I^{1} \cup\{1\}, J^{1}\right)$ and, for each $j \in N, \pi\left(l_{N} ; e\right) \geq \pi\left(l_{N}^{j} ; e^{j}\right)$, we obtain that $\left(\left|N^{\alpha}\right|+\left|N^{\beta}\right|-1\right) \pi\left(l_{N} ; e\right)-\sum_{i \in N^{\alpha}} \pi\left(l_{N}^{i} ; e^{i}\right)-\sum_{j \in N^{\beta}} \pi\left(l_{N}^{j} ; e^{j}\right) \geq 0$.

The same argument may be employed to prove the statement when $J^{m} \cup\{m\} \supseteq J^{1}$.

Putting Lemmata 8 and 9 together we obtain that following result.

Lemma 10. If $I^{1} \cup\{1\} \supseteq I^{m}$ or $J^{m} \cup\{m\} \supseteq J^{1}$ then any Individually Rational Groves mechanism runs a deficit.

Proof. If $i \in I^{1}$ or $m \in J^{m}$, then the result follows from Lemma 8. Otherwise, all the conditions of 9 are satisfied.

Proof of Proposition 3: Let $c_{\alpha} \leq c_{\beta},\left|N^{\alpha}\right| \leq\left|N^{\beta}\right|$ and assume that for some $T>0$, $\left|N^{\beta}\right| \geq \zeta(T, e)+1+\frac{c_{\alpha}}{T}+\frac{c_{\beta}}{T}$. Consider $e^{1}$. There are three possibilities regarding $J^{1}$. First, $J^{1}=\emptyset$. By Lemma 10, the statement is true. Second, $J^{1}=N^{\beta}$. Since by assumption $\left|N^{\beta}\right| c_{\beta} \geq\left|N^{\alpha}\right| c_{\alpha}$ this solution yields at most the same surplus as $\left(N^{\alpha}, \emptyset\right)$. Thus, by Lemma 8 , the statement is true. Finally, $1 \leq\left|J^{1}\right|<\left|N^{\beta}\right|$. This implies that $0 \leq\left|I^{1}\right|<\left|N^{\alpha}\right|$. As on 
both sides at least one agent is not learning from Lemma 3 it follows that $\sum_{k \in N^{\alpha} \backslash I^{1}} \theta_{k} \leq c_{\beta}$ and $\sum_{k \in N^{\beta} \backslash J^{1}} \theta_{k} \leq c_{\alpha}$, or

$$
\sum_{k \in N^{\alpha} \backslash I^{1}} \theta_{k}+\sum_{k \in N^{\beta} \backslash J^{1}} \theta_{k} \leq c_{\alpha}+c_{\beta} .
$$

Let, for each $\lambda \in\{\alpha, \beta\}$, and each $T>0, \zeta^{\lambda}(T, e)=\left|\left\{i \in N^{\lambda} \mid \theta_{i}<T\right\}\right|$. By construction, for each $T>0, \zeta^{\alpha}(T, e)=\zeta^{\alpha}\left(T, e^{1}\right)+1$. Therefore, for each $T>0$,

$$
\sum_{k \in N^{\alpha} \backslash I^{1}} \theta_{k} \geq T\left(\left|N^{\alpha} \backslash I^{1}\right|-\zeta^{\alpha}(T, e)-1\right) .
$$

Similarly, for each $T>0$,

$$
\sum_{k \in N^{\beta} \backslash J^{1}} \theta_{k} \geq T\left(\left|N^{\beta} \backslash J^{1}\right|-\zeta^{\beta}(T, e)\right) .
$$

Combining, (8.11), (8.12) and (8.13) and noting that for each $T>0, \zeta(T, e)=\zeta^{\alpha}(T, e)+$ $\zeta^{\beta}(T, e)$, we obtain

$$
\zeta(T, e)+1+\frac{c_{\alpha}}{T}+\frac{c_{\beta}}{T} \geq\left|N^{\alpha} \backslash I^{1}\right|+\left|N^{\beta} \backslash J^{1}\right| .
$$

By recasting the previous equation we obtain

$$
\left|I^{1}\right|+\left|J^{1}\right| \geq\left|N^{\alpha}\right|+\left|N^{\beta}\right|-\zeta(T, e)-\frac{c_{\alpha}}{T}-\frac{c_{\beta}}{T}-1 .
$$

By assumption, for some $T>0,\left|N^{\beta}\right| \geq \zeta(T, e)-\frac{c_{\alpha}}{T}-\frac{c_{\beta}}{T}-1$, therefore

$$
\left|I^{1}\right|+\left|J^{1}\right| \geq\left|N^{\alpha}\right| \text {. }
$$

By assumption, $c_{\alpha} \leq c_{\beta}$, therefore

$$
c_{\alpha}\left|I^{1}\right|+c_{\beta}\left|J^{1}\right| \geq c_{\alpha}\left|I^{1}\right|+c_{\alpha}\left|J^{1}\right| \geq c_{\alpha}\left|N^{\alpha}\right| .
$$

Therefore, $z^{1}\left(N^{\alpha}, \emptyset\right) \geq \pi\left(l_{N}^{1} ; e^{1}\right)$ so that, by the definition of $l_{N}^{1}, z^{1}\left(N^{\alpha}, \emptyset\right)=\pi\left(l_{N}^{1} ; e^{1}\right)$. By Lemma 8 , the statement is true.

Proof of Proposition 4: Let $c_{\alpha} \leq c_{\beta},\left|N^{\alpha}\right| \geq \frac{c_{\beta}}{c_{\alpha}}\left|N^{\beta}\right|$ and suppose that for some $T>0$, $\left|N^{\alpha}\right| \geq \frac{c_{\beta}}{c_{\alpha}}(\zeta(T, e)+1)+\frac{2 c_{\beta}}{T}$. Consider $e^{m}$. There are three possibilities regarding $I^{m}$. First, $I^{m}=\emptyset$. By Lemma 10 the statement is true. Second, $I^{m}=N^{\alpha}$. Since, by assumption, $c_{\alpha}\left|N^{\alpha}\right| \geq c_{\beta}\left|N^{\beta}\right|$ this solution yields at most the same surplus as $\left(\emptyset, N^{\beta}\right)$. Thus, by Lemma 8 , the statement is true. Finally, $1 \leq\left|I^{m}\right|<N^{\alpha}$. This implies that $0 \leq\left|J^{m}\right|<N^{\beta}$. As on both sides at least some agent is not learning, from Lemma 3 it follows that $\sum_{k \in N^{\alpha} \backslash I^{m}} \theta_{k} \leq c_{\beta}$ and $\sum_{k \in N^{\beta} \backslash J^{m}} \theta_{k} \leq c_{\alpha}$, or, since by assumption $c_{\alpha} \leq c_{\beta}$,

$$
c_{\alpha} \sum_{k \in N^{\alpha} \backslash I^{m}} \theta_{k}+c_{\beta} \sum_{k \in N^{\beta} \backslash J^{m}} \theta_{k} \leq 2 c_{\alpha} c_{\beta} .
$$

Let, for each $\lambda \in\{\alpha, \beta\}$, and each $T>0, \zeta^{\lambda}(T, e)=\left|\left\{i \in N^{\lambda} \mid \theta_{i}<T\right\}\right|$. By construction, for each $T>0, \zeta^{\beta}(T, e)=\zeta^{\beta}\left(T, e^{1}\right)+1$. Therefore, for each $T>0$,

$$
\sum_{k \in N^{\beta} \backslash J^{m}} \theta_{k} \geq T\left(\left|N^{\beta} \backslash J^{m}\right|-\zeta^{\beta}(T, e)-1\right) .
$$


Similarly, for each $T>0$,

$$
\sum_{k \in N^{\alpha} \backslash I^{m}} \theta_{k} \geq T\left(\left|N^{\alpha} \backslash I^{m}\right|-\zeta^{\alpha}(T, e)\right) .
$$

Combining, (8.18), (8.19) and (8.20) we obtain

$$
c_{\alpha} T\left(\left|N^{\alpha} \backslash I^{m}\right|-\zeta^{\alpha}(T, e)\right)+c_{\beta} T\left(\left|N^{\beta} \backslash J^{m}\right|-\zeta^{\beta}(T, e)-1\right) \leq 2 c_{\alpha} c_{\beta},
$$

and after some algebraic manipulations,

(8.22) $c_{\alpha}\left|N^{\alpha}\right|-c_{\alpha}\left(\zeta^{\alpha}(T, e)+1\right)+c_{\beta}\left|N^{\beta}\right|-c_{\beta} \zeta^{\beta}(T, e) \leq 2 \frac{c_{\alpha} c_{\beta}}{T}+c_{\alpha}\left|I^{m}\right|+c_{\beta}\left|J^{m}\right|$

Noting that for each $T>0, \zeta(T, e)=\zeta^{\alpha}(T, e)+\zeta^{\beta}(T, e)$ and that, by assumption $c_{\alpha} \leq c_{\beta}$, the expression above becomes

$$
c_{\alpha}\left|N^{\alpha}\right|-c_{\beta}(\zeta(T, e)+1)+c_{\beta}\left|N^{\beta}\right| \leq 2 \frac{c_{\alpha} c_{\beta}}{T}+c_{\alpha}\left|I^{m}\right|+c_{\beta}\left|J^{m}\right|
$$

Finally, dividing both sides by $c_{\beta}>0$ we obtain

$$
\left|N^{\alpha}\right|-\frac{c_{\beta}}{c_{\alpha}}(\zeta(T, e)+1)-2 \frac{c_{\beta}}{T}+\frac{c_{\beta}}{c_{\alpha}}\left|N^{\beta}\right| \leq\left|I^{m}\right|+\frac{c_{\beta}}{c_{\alpha}}\left|J^{m}\right|
$$

By assumption, for some $T>0,\left|N^{\alpha}\right| \geq \frac{c_{\beta}}{c_{\alpha}}(\zeta(T, e)+1)+\frac{2 c_{\beta}}{T}$, therefore

$$
\frac{c_{\beta}}{c_{\alpha}}\left|N^{\beta}\right| \leq\left|I^{m}\right|+\frac{c_{\beta}}{c_{\alpha}}\left|J^{m}\right| \text {, or } c_{\beta}\left|N^{\beta}\right| \leq c_{\alpha}\left|I^{m}\right|+c_{\beta}\left|J^{m}\right| \text {. }
$$

Therefore, $z^{m}\left(\emptyset, N^{\beta}\right) \geq \pi\left(l_{N}^{m} ; e^{m}\right)$ so that, by the definition of $l_{N}^{m}, z^{m}\left(\emptyset, N^{\beta}\right)=\pi\left(l_{N}^{m} ; e^{m}\right)$. By Lemma 8 , the statement is true.

\section{Appendix 2. Proof of Proposition 5}

Proposition 7. If a mechanism $\varphi$ satisfies Strategy-Proofness, Anonymity and Assignment Efficiency, then there exists some function $f: \mathbb{R}_{+} \rightarrow \mathbb{R}$, such that for each $e=\left(\theta_{N}, c\right) \in \mathcal{E}$, with $\left(l_{N}, t_{N}\right)=\varphi(e)$,

$$
t_{1}=\left\{\begin{array}{cc}
v_{2}\left(l_{N} ; \theta_{2}\right)+f\left(\theta_{2}\right) & \text { if } \theta_{2} \leq c \\
f\left(\theta_{2}\right) & \text { if } \theta_{2}>c
\end{array}\right.
$$

and

$$
t_{2}=\left\{\begin{array}{cl}
v_{1}\left(l_{N} ; \theta_{1}\right)+f\left(\theta_{1}\right) & \text { if } \theta_{1} \leq c \\
f\left(\theta_{1}\right)-c & \text { if } \theta_{1}>c .
\end{array}\right.
$$

Proof. The domain of preference profiles is convex (and hence smoothly connected). Once more we appeal to Holmstrom's characterization [13]. A mechanism $\varphi$ satisfies Strategy Proofness and Assignment Efficiency if and only if there exists some function $h_{i}: \mathbb{R}_{+} \rightarrow \mathbb{R}$ such that for each $e=\left(\theta_{N}, c\right) \in \mathcal{E}$, with $\left(l_{N}, t_{N}\right)=\varphi(e)$, we have $l_{N} \in \Sigma(e)$, and, for each $i \in N$,

Setting

$$
t_{i}=v_{N \backslash\{i\}}\left(l_{N} ; \theta_{N \backslash\{i\}}\right)-h_{i}\left(\theta_{N \backslash\{i\}}\right)
$$

$$
h_{i}\left(\theta_{N \backslash\{i\}}\right)=\left\{\begin{array}{cl}
-f_{i}\left(\theta_{N \backslash\{i\}}\right) & \text { if } \theta_{N \backslash\{i\}} \leq c, \\
v_{N \backslash\{i\}}\left(l_{N} ; \theta_{N \backslash\{i\}}\right)-f_{i}\left(\theta_{N \backslash\{i\}}\right) & \text { if } \theta_{N \backslash\{i\}}>c,
\end{array}\right.
$$

we obtain, for each $i \in N$, 


$$
t_{i}=\left\{\begin{array}{cl}
v_{N \backslash\{i\}}\left(l_{N} ; \theta_{N \backslash\{i\}}\right)+f_{i}\left(\theta_{N \backslash\{i\}}\right) & \text { if } \theta_{N \backslash\{i\}} \leq c \\
f_{i}\left(\theta_{N \backslash\{i\}}\right) & \text { if } \theta_{N \backslash\{i\}}>c .
\end{array}\right.
$$

Let $\theta \in\left[0, \frac{c}{2}\right)$ and $\left(l_{N}, t_{N}\right)=\varphi((\theta, \theta), c)$. By Assignment Efficiency, $l_{N}=(0,0)$. Hence, using (9.1), we obtain $u_{1}\left(\varphi\left(l_{N}, t_{N}\right)\right)=f_{1}(\theta)$ and $u_{2}\left(\varphi\left(l_{N}, t_{N}\right)\right)=f_{2}(\theta)$. By Anonymity, $u_{1}\left(\varphi\left(l_{N}, t_{N}\right)\right)=u_{2}\left(\varphi\left(l_{N}, t_{N}\right)\right)$. Hence,

$$
\text { for each } \theta \in\left[0, \frac{c}{2}\right), \quad f_{1}(\theta)=f_{2}(\theta) \text {. }
$$

Let $\theta \in\left[\frac{c}{2}, c\right]$ and $\left(l_{N}, t_{N}\right)=\varphi((\theta, \theta), c)$. By Assignment Efficiency, $l_{N}=(1,0)$. Hence, using (9.1), we obtain $u_{1}\left(\varphi\left(l_{N}, t_{N}\right)\right)=\theta-c+\left[\theta+f_{1}(\theta)\right]$ and $u_{2}\left(\varphi\left(l_{N}, t_{N}\right)\right)=\theta+\left[\theta-c+f_{2}(\theta)\right]$. By Anonymity, $u_{1}\left(\varphi\left(l_{N}, t_{N}\right)\right)=u_{2}\left(\varphi\left(l_{N}, t_{N}\right)\right)$. Hence,

$$
\text { for each } \theta \in\left[\frac{c}{2}, c\right], \quad f_{1}(\theta)=f_{2}(\theta) \text {. }
$$

Let $\theta \in(c,+\infty)$ and $\left(l_{N}, t_{N}\right)=\varphi((\theta, \theta), c)$. By Assignment Efficiency, $l_{N}=(1,0)$. Hence, using (9.1), we obtain $u_{1}\left(\varphi\left(l_{N}, t_{N}\right)\right)=\theta-c+f_{1}(\theta)$ and $u_{2}\left(\varphi\left(l_{N}, t_{N}\right)\right)=\theta+f_{2}(\theta)$. By Anonymity, $u_{1}\left(\varphi\left(l_{N}, t_{N}\right)\right)=u_{2}\left(\varphi\left(l_{N}, t_{N}\right)\right)$. Hence, for each $\theta \in(c,+\infty), f_{1}(\theta)=f_{2}(\theta)+c$.

$$
\text { for each } \theta \in(c,+\infty), \quad f_{1}(\theta)=f_{2}(\theta)+c \text {. }
$$

Setting for each $\theta \in \mathbb{R}_{+}, f(\theta)=f_{1}(\theta)$ and combining (9.1), (9.2), (9.3), (9.4), we obtain the desired result.

Let $\Phi^{g} \subset \Phi$ denote the set of Anonymous and Feasible Groves mechanisms. Appealing to Proposition 7 we may associate with any $\varphi \in \Phi^{g}$ some function $f: \mathbb{R}_{+} \rightarrow \mathbb{R}$. In addition, we may express the sum of transfers prescribed by any $\varphi \in \Phi^{g}$, at each profile, in terms of this function $f$. For each $e=\left(\left(\theta_{1}, \theta_{2}\right), c\right) \in \mathcal{E}$, with $\left(l_{N}, t_{N}\right)=\varphi(e)$, we have

$$
t_{1}+t_{2}=S^{f}\left(\theta_{1}, \theta_{2}\right)=\left\{\begin{array}{cl}
f\left(\theta_{2}\right)+f\left(\theta_{1}\right) & \text { if } \theta_{1}+\theta_{2} \leq c, \\
f\left(\theta_{2}\right)+\theta_{2}+f\left(\theta_{1}\right)+\theta_{1}-c & \text { if } \theta_{1}+\theta_{2}>c \text { and } \theta_{1}, \theta_{2} \leq c, \\
f\left(\theta_{2}\right)+\theta_{2}+f\left(\theta_{1}\right) & \text { if } \theta_{1}>c \text { and } \theta_{2} \leq c \\
f\left(\theta_{2}\right)+f\left(\theta_{1}\right)+\theta_{1}-c & \text { if } \theta_{1} \leq c \text { and } \theta_{2}>c \\
f\left(\theta_{2}\right)+f\left(\theta_{1}\right)-c & \text { if } \theta_{1}>c \text { and } \theta_{2}>c
\end{array}\right.
$$

Proposition 8. Let $\varphi \in \Phi^{g}$ be a Groves mechanism associated with some function $f: \mathbb{R}_{+} \rightarrow \mathbb{R}$. If $\varphi$ is Second Best Efficient, then for each $c>0$, each $\theta^{\prime}, \theta^{\prime \prime} \in[0, c]$, with $\theta^{\prime}<\theta^{\prime \prime}$,

$$
f\left(\theta^{\prime \prime}\right)+\theta^{\prime \prime} \geq f\left(\theta^{\prime}\right)+\theta^{\prime}
$$

Proof. Suppose not. Let there exist $\theta^{\prime}, \theta^{\prime \prime} \in[0, c]$, with $\theta^{\prime}<\theta^{\prime \prime}$, and $\epsilon>0$ such that

$$
f\left(\theta^{\prime \prime}\right)+\theta^{\prime \prime}+\epsilon=f\left(\theta^{\prime}\right)+\theta^{\prime} .
$$

Consequently, $f\left(\theta^{\prime}\right)-\left[f\left(\theta^{\prime \prime}\right)+\epsilon\right]=\theta^{\prime \prime}-\theta^{\prime}$. Since, by assumption, $\theta^{\prime \prime}-\theta^{\prime}>0$, we obtain

$$
f\left(\theta^{\prime}\right)>f\left(\theta^{\prime \prime}\right)+\epsilon
$$

Define $g: \mathbb{R}_{+} \rightarrow \mathbb{R}$ to be such that $g(\theta)=f(\theta)$, for each $\theta \in \mathbb{R}_{+} \backslash\left\{\theta^{\prime \prime}\right\}$, and $g\left(\theta^{\prime \prime}\right)=f\left(\theta^{\prime \prime}\right)+\epsilon$. 
Using (9.5) and (9.6), we obtain for each $\theta_{1} \in \mathbb{R}_{+}, S^{g}\left(\theta_{1}, \theta^{\prime \prime}\right) \leq S^{f}\left(\theta_{1}, \theta^{\prime}\right)$. Similarly, for each $\theta_{2} \in \mathbb{R}_{+}, S^{g}\left(\theta^{\prime \prime}, \theta^{2}\right) \leq S^{f}\left(\theta^{\prime}, \theta^{2}\right)$. By construction, for each $\left(\theta_{1}, \theta_{2}\right) \in \mathbb{R}_{+}^{2}$ such that $\theta_{1} \neq \theta^{\prime \prime}$ and $\theta_{2} \neq \theta^{\prime \prime}, S^{g}\left(\theta_{1}, \theta_{2}\right)=S^{f}\left(\theta_{1}, \theta_{2}\right)$. By Feasibility, for each $\left(\theta_{1}, \theta_{2}\right) \in \mathbb{R}_{+}^{2}, S^{f}\left(\theta_{1}, \theta_{2}\right) \leq$ 0 . Therefore, for each $\left(\theta_{1}, \theta_{2}\right) \in \mathbb{R}_{+}^{2}, S^{g}\left(\theta_{1}, \theta_{2}\right) \leq 0$. Let $\varphi^{\prime}$ be some Anonymous Groves mechanism that agrees with $\varphi$ on the linguistic assignment on all economies and, moreover, is associated with $g$. The mechanism $\varphi^{\prime}$ satisfies Feasibility and, thus, $\varphi^{\prime} \in \Phi$.

Finally, by construction, $\varphi^{\prime}$ Pareto dominates $\varphi$ at each profile $\left(\theta_{1}, \theta_{2}\right) \in\left\{\left(\theta_{1}, \theta_{2}\right) \in \mathbb{R}_{+}^{2}\right.$ : either $\theta_{1}=\theta^{\prime \prime}$ or $\theta_{2}=\theta^{\prime \prime}$ or both $\}$, while $\varphi^{\prime}$ coincides with $\varphi$ otherwise. Hence, $\varphi^{\prime}$ Pareto dominates $\varphi$, a contradiction.

Lemma 11. Let $\varphi \in \Phi^{g}$ be a Groves mechanism associated with some function $f: \mathbb{R}_{+} \rightarrow \mathbb{R}$. If $\varphi$ is Second Best Efficient then, for each $\theta>c, f(\theta)=\frac{c}{2}$.

Proof. Suppose first, by way of contradiction, that for some $\tilde{\theta}>c, f(\tilde{\theta})>\frac{c}{2}$. By Proposition 7 , letting $\left(l_{N}, t_{N}\right)=\varphi((\tilde{\theta}, \tilde{\theta}), c), S^{f}(\tilde{\theta}, \tilde{\theta})=2 f(\tilde{\theta})-c$. By assumption, $f(\tilde{\theta})>\frac{c}{2}$, therefore $S^{f}(\tilde{\theta}, \tilde{\theta})>0$, which contradicts Feasibility.

Suppose then that for some $\tilde{\theta}>c, f(\tilde{\theta})<\frac{c}{2}$. In particular, let $f(\tilde{\theta})=\frac{c}{2}-\epsilon$, for some $\epsilon>0$. Consider first profile $(\theta, \tilde{\theta})$, for some $\theta \in[0, c]$. By Proposition 7 , letting $\left(l_{N}, t_{N}\right)=\varphi((\theta, \tilde{\theta}), c)$, $S^{f}(\theta, \tilde{\theta})=\frac{c}{2}-\epsilon+f(\theta)+\theta-c$. By Proposition 8 ,

$$
S^{f}(\theta, \tilde{\theta})=\frac{c}{2}-\epsilon+f(\theta)+\theta-c \leq \frac{c}{2}-\epsilon-c+[f(c)+c]=\frac{c}{2}-\epsilon+f(c) .
$$

Note that, by Feasibility, $f(c) \leq-\frac{c}{2}$. Hence,

$$
S^{f}(\theta, \tilde{\theta})=\frac{c}{2}-\epsilon+f(c) \leq \frac{c}{2}-\epsilon-\frac{c}{2}=-\epsilon<0 .
$$

Consider, finally, the profile $(\theta, \tilde{\theta})$, for some $\theta \in(c,+\infty)$. Letting again $\left(l_{N}, t_{N}\right)=\varphi((\theta, \tilde{\theta}), c)$, and appealing to Proposition 7, we obtain $S^{f}(\theta, \tilde{\theta})=\frac{c}{2}-\epsilon+f(\theta)-c$. We have already established earlier in the proof that $f(\theta) \leq \frac{c}{2}$. Hence, $S^{f}(\theta, \tilde{\theta}) \leq \frac{c}{2}-\epsilon+\frac{c}{2}-c=-\epsilon<0$. Hence, for each $\theta \in \mathbb{R}_{+}, t_{1}+t_{2}<0$, which contradicts Condition $A$, a necessary condition for Second-Best Efficiency.

Lemma 12. Let $\varphi \in \Phi^{g}$ be a Groves mechanism associated with some function $f: \mathbb{R}_{+} \rightarrow \mathbb{R}$. For each $\theta \in\left(\frac{c}{2}, c\right], f(\theta) \leq \frac{c}{2}-\theta$.

By Proposition $7, S^{f}(\theta, \theta)=2 f(\theta)+2 \theta-c$. By Feasibility, $S^{f}(\theta, \theta) \leq 0$. Hence, $f(\theta) \leq \frac{c}{2}-\theta$.

Lemma 13. Let $\varphi \in \Phi^{g}$ be a Groves mechanism associated with some function $f: \mathbb{R}_{+} \rightarrow \mathbb{R}$. If $\varphi$ is Second Best Efficient then, for each $\theta \in\left[0, \frac{c}{2}\right], f(\theta)=0$.

Proof. Suppose, by way of contradiction, that there exists some $\tilde{\theta} \in\left[0, \frac{c}{2}\right]$ such that $f(\tilde{\theta}) \neq 0$. If $f(\tilde{\theta})>0$ we would obtain $S^{f}(\tilde{\theta}, \tilde{\theta})=2 f(\tilde{\theta})>0$, a violation of Feasibility. Therefore, it must be $f(\tilde{\theta})<0$.

Step 1. Let $\theta \in(c,+\infty)$. By Lemma 7 , using the fact that $\tilde{\theta} \in\left[0, \frac{c}{2}\right]$ and $\theta \in(c,+\infty)$, we obtain

$$
S^{f}(\tilde{\theta}, \theta)=f(\theta)+f(\tilde{\theta})+\tilde{\theta}-c .
$$


By Lemma 11, using the fact that $\theta \in(c,+\infty)$,

$$
S^{f}(\tilde{\theta}, \theta)=\frac{c}{2}+f(\tilde{\theta})+\tilde{\theta}-c=-\frac{c}{2}+\tilde{\theta}+f(\tilde{\theta}) .
$$

By assumption, $f(\tilde{\theta})<0$ and $-\frac{c}{2}+\tilde{\theta} \leq 0$, hence

$$
\text { for each } \theta \in(c,+\infty), S^{f}(\tilde{\theta}, \theta)<0 \text {. }
$$

Step 2. Let $\theta \in\left[0, \frac{c}{2}\right]$. By Proposition 7 , using the fact that $\tilde{\theta}, \theta \in\left[0, \frac{c}{2}\right]$, we obtain

$$
S^{f}(\tilde{\theta}, \theta)=f(\theta)+f(\tilde{\theta})
$$

and, in particular $S^{f}(\theta, \theta)=2 f(\theta)$. By Feasibility, $S^{f}(\theta, \theta) \leq 0$. Hence, $f(\theta) \leq 0$. This latter fact, combined with the assumption that $f(\tilde{\theta})<0$, yields

$$
\text { for each } \theta \in\left[0, \frac{c}{2}\right], S^{f}(\tilde{\theta}, \theta)<0 \text {. }
$$

Step 3. Let $\theta \in\left(\frac{c}{2}, c\right]$. By Proposition 7 , using the fact that $\tilde{\theta} \in\left[0, \frac{c}{2}\right]$ and $\theta \in\left(\frac{c}{2}, c\right]$, we obtain

$$
S^{f}(\tilde{\theta}, \theta)=f(\theta)+\theta+f(\tilde{\theta})+\tilde{\theta}-c .
$$

By Lemma 12, substituting for $f(\theta)$, we obtain

$$
S^{f}(\tilde{\theta}, \theta) \leq f(\tilde{\theta})+\tilde{\theta}-\frac{c}{2}
$$

By assumption, $f(\tilde{\theta})<0$ and $\tilde{\theta} \in\left[0, \frac{c}{2}\right]$, hence

$$
\text { for each } \theta \in\left(\frac{c}{2}, c\right], S^{f}(\tilde{\theta}, \theta)<0 \text {. }
$$

Step 3. From Steps 1-3 we obtain that for each $\theta \in \mathbb{R}_{+}, S^{f}(\tilde{\theta}, \theta)<0$. This constitutes a violation of Condition A, a necessary condition for Second-Best Efficiency.

Lemma 14. Let $\varphi \in \Phi$ be a Groves mechanism associated with some function $f: \mathbb{R}_{+} \rightarrow \mathbb{R}$. If $\varphi$ is Second Best Efficient then, for each $\theta \in\left(\frac{c}{2}, c\right], f(\theta)=\frac{c}{2}-\theta$.

Proof. Suppose, by way of contradiction, that there exist some $\tilde{\theta} \in\left(\frac{c}{2}, c\right]$ such that $f(\tilde{\theta}) \neq \frac{c}{2}-\tilde{\theta}$. Lemma 12 rules out $f(\tilde{\theta})>\frac{c}{2}-\tilde{\theta}$. Therefore, it must be $f(\tilde{\theta})<\frac{c}{2}-\tilde{\theta}$.

Step 1. Let $\theta \in(c,+\infty)$. By Proposition 7 , using the fact that $\tilde{\theta} \in\left(\frac{c}{2}, c\right]$ and $\theta \in(c,+\infty)$, we obtain

$$
S^{f}(\tilde{\theta}, \theta)=f(\theta)+f(\tilde{\theta})+\tilde{\theta}-c .
$$

By Lemma 11, using the fact that $\theta \in(c,+\infty)$ and substituting for $f(\theta)$, this becomes

$$
S^{f}(\tilde{\theta}, \theta)=f(\tilde{\theta})+\tilde{\theta}-\frac{c}{2} .
$$

By assumption, $f(\tilde{\theta})<\frac{c}{2}-\tilde{\theta}$, hence

$$
\text { for each } \theta \in(c,+\infty), S^{f}(\tilde{\theta}, \theta)<0 .
$$

Step 2. Let $\theta \in[0, c]$ and $\tilde{\theta}+\theta \leq c$. By Proposition 7 ,

$$
S^{f}(\tilde{\theta}, \theta)=f(\theta)+f(\tilde{\theta}) .
$$


Since $\tilde{\theta}+\theta \leq c$ and $\tilde{\theta} \in\left(\frac{c}{2}, c\right]$ it must be $\theta<\frac{c}{2}$. Therefore, by Lemma 13, $f(\theta)=0$. Consequently, $S^{f}(\tilde{\theta}, \theta)=f(\tilde{\theta})$. By assumption, $f(\tilde{\theta})<\frac{c}{2}-\tilde{\theta}$ and $\tilde{\theta}>\frac{c}{2}$. Hence,

$$
\text { if } \tilde{\theta}+\theta \leq c \text {, then for each } \theta \in[0, c], S^{f}(\tilde{\theta}, \theta)<0 .
$$

Step 3. Let $\theta \in[0, c]$ and $\tilde{\theta}+\theta>c$. By Proposition 7,

$$
S^{f}(\tilde{\theta}, \theta)=f(\theta)+\theta+f(\tilde{\theta})+\tilde{\theta}-c .
$$

By assumption, $f(\tilde{\theta})<\frac{c}{2}-\tilde{\theta}$. Hence, substituting for $f(\tilde{\theta})$ we obtain

$$
S^{f}(\tilde{\theta}, \theta)<\theta+f(\theta)-\frac{c}{2}
$$

Since $\theta \in[0, c]$, by Proposition $8, f(c)+c \geq f(\theta)+\theta$. Substituting for $f(\theta)+\theta$ we obtain $S^{f}(\tilde{\theta}, \theta)<\frac{c}{2}+f(c)$. By Lemma $12, f(c) \leq-\frac{c}{2}$, so that

$$
\text { if } \tilde{\theta}+\theta>c \text {, then for each } \theta \in[0, c], S^{f}(\tilde{\theta}, \theta)<0 \text {. }
$$

Step 4. From Steps 1-3 we obtain that for each $\theta \in \mathbb{R}_{+}, S^{f}(\tilde{\theta}, \theta)<0$. This constitutes a violation of Condition A, a necessary condition for Second-Best Efficiency.

The function $f$ corresponding to the SBM mechanism is

$$
f(\theta)=\left\{\begin{array}{cc}
0 & \text { if } \theta \leq \frac{c}{2} \\
\frac{c}{2}-\theta & \text { if } \frac{c}{2}<\theta \leq c \\
\frac{c}{2} & \text { if } \theta>c
\end{array}\right.
$$

The preceding results prove the only if part of Proposition 5. Let us then prove that the SBM is indeed Second Best Efficient.

Lemma 15. If a mechanism $\varphi$ is Strategy-Proof, then for each profile $\left(\left(\theta_{1}, \theta_{2}\right), c\right) \in \mathcal{E}$ there exists $\left(p_{1}, p_{2}\right) \leq\left(\theta_{1}, \theta_{2}\right)$ such that for each $i \in\{1,2\}$,

- if $l_{i}=0$, letting $\left(l_{N}(x), t_{N}(x)\right)=\varphi\left(\left(x, \theta_{N \backslash\{i\}}\right), c\right)$, then for each $x \leq \theta_{i}, l_{i}(x)=l_{i}=0$ and $t_{i}(x)=t_{i}(0)$,

- if $l_{i}=1$, letting $\left(l_{N}(x), t_{N}(x)\right)=\varphi\left(\left(x, \theta_{N \backslash\{i\}}\right), c\right)$, then for each $x \geq p_{i}, l_{i}(x)=l_{i}=1$ and $t_{i}(x)=-p_{i}+t_{i}(0)$.

This lemma is implicit in Holmström [13]. An explicit proof can be found in Nisan et al [19]. We omit the proof.

Lemma 16. If a mechanism $\varphi^{\prime} \in \Phi$ Pareto dominates the $S B M$, then $\varphi^{\prime} \in \Phi$ coincides with the $S B M$ at profiles $\left(\theta_{1}, \theta_{2}\right) \in \mathbb{R}_{+}^{2}$ such that either $\theta_{1}, \theta_{2} \geq \frac{c}{2}$ or $\theta_{1}, \theta_{2} \leq \frac{c}{2}$.

Proof.

This follows straightforwardly from the fact that the SBM prescribes an efficient assignment and a vector of transfers that sums up to zero at those profiles.

Proposition 9. There does not exist a mechanism $\varphi^{\prime} \in \Phi$ that Pareto dominates the SBM. 
By way of contradiction, suppose that some $\varphi^{\prime} \in \Phi$ Pareto dominates the SBM. By Lemma 16 if $\varphi^{\prime}$ Pareto dominates SBM it must be so for some profile $\left(\theta_{1}, \theta_{2}\right) \in\left[0, \frac{c}{2}\right) \times\left(\frac{c}{2},+\infty\right)$. By Anonymity that will suffice, and we do not need to concern ourselves with $\left(\theta_{1}, \theta_{2}\right) \in$ $\left.\left(\frac{c}{2},+\infty\right) \times\left[0, \frac{c}{2}\right) ?\right)$. Define for each $c>0$, each $\theta_{1} \in\left[0, \frac{c}{2}\right)$ and each $x \geq 0$,

$$
\left(l_{N}^{\theta_{1}}(x), t_{N}^{\theta_{1}}(x)\right)=\varphi^{\prime}\left(\left(\theta_{1}, x\right), c\right) .
$$

Step 1. Suppose that there exists $\tilde{e}=\left(\left(\tilde{\theta_{1}}, \tilde{\theta_{2}}\right), c\right) \in\left[0, \frac{c}{2}\right) \times\left(\frac{c}{2},+\infty\right) \times \mathbb{R}_{+}$, with $\tilde{\theta_{1}}+\tilde{\theta_{2}}>c$, such that $\left.((0,0)), \tilde{t}_{N}\right)=\varphi^{\prime}(e)$. By Strategy-Proofness and Lemma $15, \tilde{t}_{2}=t_{2}^{\tilde{\theta}_{1}}(0)$. By Lemma $16, t_{2}^{\tilde{\theta}_{1}}(0)=0$, the transfer the SBM prescribes at any economy $\left(\left(\tilde{\theta}_{1}, 0\right), c\right) \in \mathcal{E}$, with $\tilde{\theta}_{1} \in\left[0, \frac{c}{2}\right)$. Hence, we obtain

$$
u_{2}\left(\varphi^{S B M}(\tilde{e})\right)>u_{2}\left(\varphi^{\prime}(\tilde{e})\right)=0 .
$$

This contradicts the fact that $\varphi^{\prime}$ Pareto dominates the SBM.

Step 2. Suppose that there exists $e=\left(\left(\theta_{1}, \theta_{2}\right), c\right) \in\left[0, \frac{c}{2}\right) \times\left(\frac{c}{2},+\infty\right) \times \mathbb{R}_{+}$, with $\theta_{1}+\theta_{2}>c$, such that $\left.((1,0)), t_{N}\right)=\varphi^{\prime}(e)$. By Lemma 15 there exists $p \in\left[0, \theta_{2}\right]$ such that

- if $x<p$, then $l_{N}^{\theta_{1}}(x)=(0,0)$ and $t_{2}^{\theta_{1}}(x)=t_{2}^{\theta_{1}}(0)$, and

- if $x \geq p$, then $l_{N}^{\theta_{1}}(x)=(1,0)$ and $t_{2}^{\theta_{1}}(x)=-p+t_{2}^{\theta_{1}}(0)$.

By Lemma 16, using the same argument as in step $1, t_{2}^{\theta_{1}}(0)=0$. If $p>c-\theta_{1}$, at any profile $\left(\theta_{1}, \theta_{2}^{\prime}\right)$, with $\theta_{2}^{\prime}>\theta_{2}$,

$$
u_{2}\left(\varphi^{S B M}\left(\left(\theta_{1}, \theta_{2}^{\prime}\right), c\right)\right)=\theta_{2}^{\prime}+\theta_{1}-c>u_{2}=\left(\varphi^{\prime}\left(\left(\theta_{1}, \theta_{2}^{\prime}\right), c\right)\right)=\theta_{2}^{\prime}-p .
$$

This contradicts the fact that $\varphi^{\prime}$ Pareto dominates the SBM.

Suppose then that $p<c-\theta_{1}$. By Lemma 15, there exists some economy $e^{\prime \prime}=\left(\left(\theta_{1}, \theta_{2}^{\prime \prime}\right), c\right) \in$ $\left[0, \frac{c}{2}\right) \times\left(p, c-\theta_{1}\right) \times \mathbb{R}_{+}$, such that $\left((1,0), t_{N}^{\prime \prime}\right)=\varphi^{\prime}\left(e^{\prime \prime}\right)$. By Lemma 16 and Strategy-Proofness, we obtain $t_{2}^{\prime \prime}=\frac{c}{2}$. Therefore,

$$
u_{1}\left(\varphi^{\prime}\left(\left(\theta_{1}, \theta_{2}^{\prime \prime}\right), c\right)\right)=\theta_{1}-\frac{c}{2}
$$

Moreover,

$$
u_{1}\left(\varphi^{S B M}\left(\left(\theta_{1}, \theta_{2}^{\prime \prime}\right), c\right)\right)=\frac{c}{2}-\theta_{2}^{\prime \prime} .
$$

By construction, $\theta_{1}+\theta_{2}^{\prime \prime}<c$. A simple algebraic manipulation over this inequality yields

$$
\theta_{1}-\frac{c}{2}<\frac{c}{2}-\theta_{2}^{\prime \prime}
$$

which implies that

$$
u_{1}\left(\varphi^{S B M}\left(\left(\theta_{1}, \theta_{2}^{\prime \prime}\right), c\right)\right)>u_{1}\left(\varphi^{\prime}\left(\left(\theta_{1}, \theta_{2}^{\prime \prime}\right), c\right)\right)
$$

This contradicts the fact that $\varphi^{\prime}$ Pareto dominates the SBM.

Step 3. Suppose that there exists $e=\left(\left(\theta_{1}, \theta_{2}\right), c\right) \in\left[0, \frac{c}{2}\right) \times\left(\frac{c}{2},+\infty\right) \times \mathbb{R}_{+}$, with $\theta_{1}+\theta_{2}>c$, such that $\left.((0,1)), t_{N}\right)=\varphi^{\prime}(e)$. Applying the same reasoning as before, for some $p \geq \frac{c}{2}$ and some $c^{+} \geq c$, we have $u_{2}\left(\varphi^{\prime}(e)\right)=\theta_{2}-c^{+}-p$. We obtain

$$
u_{2}\left(\varphi^{S B M}(e)\right)=\theta_{1}+\theta_{2}-c>u_{2}\left(\varphi^{\prime}(e)\right) .
$$


This contradicts the fact that $\varphi^{\prime}$ Pareto dominates the SBM.

Combining steps 1 to 3 , the proof is complete. 